\title{
Modelling solar irradiance from ground-based photometric observations
}

\author{
Theodosios Chatzistergos ${ }^{1,2, *}$, Ilaria Ermolli ${ }^{1}$, Fabrizio Giorgi $^{1}$, Natalie A. Krivova ${ }^{2}$, \\ and Cosmin Constantin Puiu ${ }^{1}$ \\ ${ }^{1}$ INAF Osservatorio Astronomico di Roma, Via Frascati 33, 00078 Monte Porzio Catone, Italy \\ ${ }^{2}$ Max Planck Institute for Solar System Research, Justus-von-Liebig-Weg 3, 37077 Göttingen, Germany
}

Received 31 May 2020 / Accepted 24 August 2020

\begin{abstract}
Total solar irradiance (TSI) has been monitored from space since 1978, i.e. for about four solar cycles. The measurements show a prominent variability in phase with the solar cycle, as well as fluctuations on timescales shorter than a few days. However, the measurements were done by multiple and usually relatively short-lived missions. The different absolute calibrations of the individual instruments and the unaccounted for instrumental trends make estimates of the possible long-term trend in the TSI highly uncertain. Furthermore, both the variability and the uncertainty are strongly wavelength-dependent. While the variability in the UV irradiance is clearly in-phase with the solar cycle, the phase of the variability in the visible range has been debated. In this paper, we aim at getting an insight into the long-term trend of TSI since 1996 and the phase of the solar irradiance variations in the visible part of the spectrum. We use independent ground-based full-disc photometric observations in Ca II K and continuum from the Rome and San Fernando observatories to compute the TSI since 1996. We follow the empirical San Fernando approach based on the photometric sum index. We find a weak declining trend in the TSI of $-7.8_{-0.8}^{+4.9} \times 10^{-3} \mathrm{Wm}^{-2} \mathrm{y}^{-1}$ between the 1996 and 2008 activity minima, while between 2008 and 2019 the reconstructed TSI shows no trend to a marginally decreasing (but statistically insignificant) trend of $-0.1_{-0.02}^{+0.25} \times 10^{-3} \mathrm{Wm}^{-2} \mathrm{y}^{-1}$. The reference TSI series used for the reconstruction does not significantly affect the determined trend. The variation in the blue continuum $(409.2 \mathrm{~nm})$ is rather flat, while the variation in the red continuum $(607.1 \mathrm{~nm})$ is marginally in anti-phase, although this result is extremely sensitive to the accurate assessment of the quiet Sun level in the images. These results provide further insights into the long-term variation of the TSI. The amplitude of the variations in the visible is below the uncertainties of the processing, which prevents an assessment of the phase of the variations.
\end{abstract}

Keywords: sun / variability / solar activity / spectral irradiance / total irradiance

\section{Introduction}

The solar irradiance (SI) is an important parameter in studies of the solar variability and Earth's climate. The spectrallyresolved SI, called the spectral solar irradiance (SSI), is defined as the solar radiative energy flux per unit area and wavelength as measured at the top of the Earth's atmosphere at a mean distance of one astronomical unit. The total solar irradiance (TSI) is the SSI integrated over the whole spectrum. Current knowledge of SI comes from space-based measurements and SI models.

Measurements collected since 1978 by a series of instruments show that the TSI varies at all discernible timescales from

\footnotetext{
*Corresponding author: chatzistergos@mps.mpg.de
}

minutes to decades. Most prominent are a clear change by $\sim 0.1 \%$ in phase with the solar cycle (Kopp, 2016) and fluctuations by up to $0.2-0.3 \%$ on timescales shorter than a few days (Domingo et al., 2009).

On timescales longer than the solar cycle, TSI changes are rather uncertain due to the uncertainties in instrumental calibrations and the limited duration of the individual space experiments. In particular, there are currently four composite records of TSI measurements, presented by Willson (1997), Dewitte et al. (2004), Fröhlich (2006), and Dudok de Wit et al. (2017), showing partly conflicting secular trends over the last four solar cycle minima.

The TSI is the integral over the entire solar radiation spectrum that ranges from X-rays to radio waves. The bulk of solar radiant energy is in the visible (Vis, 400-700 $\mathrm{nm}$ ), followed 
by the infrared (IR, $700 \mathrm{~nm}^{-1} \mathrm{~mm}$ ) and ultraviolet (UV, 100$400 \mathrm{~nm}$ ) bands. Measurements of the SSI also started in the 1970 s, but are less continuous and more uncertain than those of TSI. Until just less than 20 years ago, they mainly covered the UV band (see Ermolli et al., 2013, for a review). Nevertheless, all present data clearly show that the form and amplitude of SSI variations are strongly wavelength dependent. Although the various experiments produced different estimates of the magnitude of UV changes, all available data agree on that they are in phase with the solar cycle (e.g. Floyd et al., 2003; Rottman et al., 2006). In contrast, SSI measurements at the IR and longer wavelengths show variations that are in anti-phase with the solar cycle. With regard to Vis, conflicting results from two experiments make SSI variations at this spectral range still uncertain. In particular, measurements from the Spectral Irradiance Monitor (SIM) on-board the SOlar Radiation and Climate Experiment (SORCE) satellite show Vis changes that are in anti-phase relation with the solar cycle (Harder et al., 2009), while those from the Variability of solar IRadiance and Gravity Oscillations (VIRGO) experiment on-board the SOlar and Heliospheric Observatory ( $\mathrm{SOHO}$ ) display an in-phase variation (Wehrli et al., 2013). However, it has been suggested that the SORCE/SIM data before 2010, which are the ones showing the anti-phase relation, are unreliable due to potential instrumental issues (Haberreiter et al., 2017; Mauceri et al., 2018, 2020).

Models that ascribe variations in SI at timescales greater than a day to solar surface magnetism have proved to be successful in reproducing existing measurements of TSI and of SSI at given spectral ranges and timescales (e.g. Domingo et al., 2009; Shapiro et al., 2017; Yeo et al., 2014, 2017b). There are various SI models presented in the literature. They can be grouped in the so-called proxy (e.g. Hudson et al., 1982; Chapman et al., 2013; Georgieva et al., 2015; Tebabal et al., 2015; Yeo et al., 2017a; Lean, 2018; Choudhary et al., 2020), semi-empirical (e.g. Fligge et al., 2000; Ermolli, 2001; Krivova et al., 2003; Crouch et al., 2008; Shapiro et al., 2010; Fontenla et al., 2011; Bolduc et al., 2012; Yeo et al., 2014; Wu et al., 2018), and physical (Yeo et al., 2017b). The proxy models use linear combinations of indices of solar magnetic features and regression to actual TSI measurements, while semi-empirical and physical models reconstruct the SI variations by employing results from spatially-resolved full-disc observations and from radiative transfer calculations performed on either atmosphere models or outcomes from magnetohydrodynamic simulations of the solar atmosphere, respectively.

Results from the various SI models show considerable disagreement in the long-term evolution of TSI with estimated changes since the Maunder minimum ranging from $0.7 \mathrm{Wm}^{-2}$ to $6 \mathrm{Wm}^{-2}$ (Wang et al., 2005; Krivova et al., 2007, 2010; Tapping et al., 2007; Steinhilber et al., 2009; Shapiro et al., 2011; Vieira et al., 2011; Judge et al., 2012, 2020; Egorova et al., 2018; Wu et al., 2018; Lockwood \& Ball, 2020). The various SI models also estimate different SSI variations on timescales longer than a few solar rotations (see Ermolli et al., 2013, for a review of this topic). In particular, for the SSI in the Vis range, most models suggest an in-phase variation with the solar cycle (e.g. Krivova et al., 2006; Ball et al., 2011; Unruh et al., 2012; Yeo et al., 2014, 2017a; Mauceri et al., 2019), although there are also models indicating an anti-phase change (e.g. Preminger et al., 2011; Fontenla et al., 2011; Fontenla \& Landi, 2018). In the UV, all SI models return variability in phase with the solar cycle.

Long and reliable time series of TSI and SSI are urgently needed to allow accurate quantification of solar contribution to the evolution of Earth's climate. To improve our understanding of the solar-cycle and long-term variability of SI, here we analyse the TSI and SSI variations in two spectral intervals in the Vis over the last two solar cycles derived from a careful analysis of two independent series of ground-based solar observations. In particular, we exploit the two longest timeseries of modern full-disc photometric observations of the solar atmosphere, performed at the Rome and San Fernando observatories, and process these data with the method developed by Chatzistergos et al. (2018b, 2019b, 2020a) to reconstruct the TSI and SSI variations over the period 1996-2020 by applying the proxy model by Chapman et al. (2013).

The paper is structured as follows. In Section 2, we describe the various full-disc solar observations and irradiance series used in our study, as well as the methods employed to process the images and to reconstruct SI variations. In Section 3, we analyse the solar cycle variability in different spectral bands covered by the full-disc observations and their phase relations. We present our results for the long-term variation of TSI over the last three solar activity minima (Sect. 4). We discuss the results in Section 5, before summarising them and drawing our conclusions in Section 6.

\section{Data and methods}

\subsection{Photometric sums and irradiance modelling}

We follow the approach by Chapman et al. (2012, 2013), who used an empirical model based on the so-called photometric sums derived from $\mathrm{Ca}$ II $\mathrm{K}$ and continuum images. The assumption is that the irradiance variability is due to the modulation by dark and bright features, such as sunspots and faculae (in white light). Faculae are much better seen in chromospheric Ca II K observations, where they have a higher contrast than in photospheric observations and are called plage. We will refer to both faculae and plage as faculae, independently of the type of their observations. Sunspots have a higher contrast in the VIS and IR and therefore images in the red continuum in the 600 $700 \mathrm{~nm}$ range are used to describe their effect. Faculae are much better represented by $\mathrm{Ca}$ II K observations (see e.g. Skumanich et al., 1984).

The photometric sum is defined as $\Sigma=\sum_{i}\left(C_{i} I_{i}^{\mathrm{CLV}}\right)$. Here $C_{i}$ is the contrast value of pixel $i$ defined as $C_{i}=\left(I_{i}-I_{i}^{\mathrm{QS}}\right) / I_{i}^{\mathrm{QS}}$, with $I_{i}$ being the intensity value of this pixel, and $I_{i}^{\mathrm{QS}}$ the intensity of the quiet Sun (QS) regions. $I_{i}^{\mathrm{CLV}}$ is the average QS centre-to-limb variation (CLV) normalised such that at the disc centre it has the value of unity. Thus $C_{i}$ is dimensionless, and the photometric sum of quiet sun regions is equal to 0 . By normalising $I_{i}^{\mathrm{CLV}}$ to unity we render the photometric sums derived from $\mathrm{Ca}$ II $\mathrm{K}$ and continuum observations analysed here directly comparable. We note, that the photometric sums as defined here depend on the pixel scale of the images. The images and their processing are described in the next section. 
Table 1. List of datasets analysed in this study.

\begin{tabular}{|c|c|c|c|c|c|c|c|c|}
\hline Observatory & Acronym & $\begin{array}{l}\text { Exposures } \\
\text { \#of frames }\end{array}$ & $\begin{array}{c}\text { Wavelength } \\
{[\mathrm{nm}]}\end{array}$ & $\begin{array}{l}\mathrm{SW} \\
{[\mathrm{nm}]}\end{array}$ & Period & $\begin{array}{l}\text { Pixel scale } \\
\text { ["/pixel] }\end{array}$ & Images & Ref. \\
\hline \multirow[t]{2}{*}{ Rome PSPT } & \multirow[t]{2}{*}{$\mathrm{RP}$} & \multirow[t]{2}{*}{25} & 393.4 & 0.25 & 1996-2020 & 2.0 & 3513 & 1 \\
\hline & & & 607.1 & 0.5 & 1997-2020 & 2.0 & 4135 & 1 \\
\hline \multirow[t]{2}{*}{ Rome PSPT } & \multirow[t]{2}{*}{ RPS } & \multirow[t]{2}{*}{1} & 393.4 & 0.25 & $2000-2020$ & 2.0 & 4230 & 1 \\
\hline & & & 607.1 & 0.5 & 2000-2020 & 2.0 & 3766 & 1 \\
\hline San Fernando CFDT2 & SF & 2 & 393.4 & 0.9 & 1996-2013 & 2.6 & 3380 & 2 \\
\hline
\end{tabular}

Notes. Columns are: name of the observatory, abbreviation used in this study, number of summed frames, central wavelength, spectral width, and period of observations, average pixel scale of the images, total number of available images, and the bibliography entry.

References. (1) Ermolli et al. (2007); (2) Chapman et al. (2013).

The TSI is calculated as a linear combination of the photometric sums in the red, $\Sigma_{\mathrm{r}}$, or blue, $\Sigma_{\mathrm{b}}$, continuum, and the one in the Ca II K line, $\Sigma_{\mathrm{k}}$ (Chapman et al., 2013):

$$
\operatorname{TSI}(t)=a\left(1+b \Sigma_{\mathrm{r}, \mathrm{b}}(t)+c \Sigma_{\mathrm{k}}(t)\right) .
$$

The three parameters, $a, b$, and $c$ of the model are determined through a linear regression of the photometric sums to the actual TSI measurements described in Section 2.3.

\subsection{Full-disc observations}

We analysed full-disc solar observations acquired with the Precision Solar Photometric Telescope at the INAF Osservatorio Astronomico di Roma (Rome/PSPT, hereafter) and with the Cartesian Full-Disk Telescope 2 at the San Fernando observatory (SFO/CFDT2, hereafter). Table 1 lists key characteristics of the analysed full-disc observations. In particular, we considered Rome/PSPT observations taken in the Ca II K line at $393.37 \mathrm{~nm}(0.25 \mathrm{~nm}$ bandwidth), blue continuum at $409.20 \mathrm{~nm}$ ( $0.25 \mathrm{~nm}$ bandwidth; blue hereafter), as well as in the red continuum at $607.1 \mathrm{~nm}(0.5 \mathrm{~nm}$ bandwidth; red hereafter). The SFO/CFDT2 filtergrams were acquired in the Ca II K line at $393.37 \mathrm{~nm}$ (0.9 nm bandwidth).

Regular monitoring of the solar atmosphere in Ca II K and the blue continuum with Rome/PSPT ${ }^{1}$ started in May 1996. Observations in the red continuum started in September 1997 (Ermolli et al., 1998, 2007). Two significant instrumental changes occurred in August 1997 and September 2001, involving the replacement of the CCD device and the $\mathrm{Ca}$ II K filter, and the telescope relocation from the Monte Mario to Monte Porzio Catone site, respectively. These changes introduced slight discontinuities in the series of the Rome/PSPT observations, which seem not to significantly affect the high photometric accuracy of the Rome/PSPT data (Ermolli et al., 2011; Chatzistergos et al., 2019b). The observations are stored as $1024 \times 1024$ pixel $^{2}$ images with $2^{\prime \prime} /$ pixel size. They are available as single exposure images (RPS, hereafter), as well as the sum of 25 exposures (RP, hereafter). The RPS data have better spatial resolution, while the RP images have an improved photometric accuracy. In this study, we considered the RP data obtained from 16 May 1996 to 12 June 2020, and the RPS images acquired from 21 August 2000 to 12 June 2020. Even though RP images are sums of 25 exposures, individual exposures were not stored before the 21st of August of 2000.

\footnotetext{
${ }^{1}$ Available at https://www.oa-roma.inaf.it/pspt-in-rome/
}

The SFO/CFDT2 observations ${ }^{2}$ analysed in this study cover the period 1996-2013. Regular solar monitoring started at the San Fernando Observatory with the Cartesian Full Disk Telescope 1 (SFO/CFDT1) in 1986. This telescope produces $512 \times 512 \mathrm{pixel}^{2}$ images with $\sim 5.1^{\prime \prime} /$ pixel size. A second telescope, SFO/CFDT2, was introduced in 1992 (Chapman et al., 2013) providing higher spatial resolution data. The images acquired with SFO/CFDT2 have dimensions $1024 \times 1024 \mathrm{pixel}^{2}$ with $\sim 2.6^{\prime \prime} /$ pixel size. The images are the sum of two exposures taken roughly $7.5 \mathrm{~min}$ apart (Chapman et al., 2013). Here, we restricted our analysis to the SFO/CFDT2 data (SF, hereafter), because of their higher resolution compared to SFO/CFDT1, as well as potential instrumental issues with SFO/CFDT1 images (Chatzistergos et al., 2020a). We also restricted our analysis to SFO/CFDT2 data taken since 1996 due to the availability of the Rome/PSPT blue continuum observations.

All observations were calibrated by the observing teams for the instrumental response. We have processed the images with the methods described by Chatzistergos et al. (2016, 2018a,b, 2019a,b,c, 2020a,b, hereafter referred to as "our"). This allowed us to consistently compensate the RP, RPS, and SF data for the intensity CLV of the QS regions and to account for any artificial intensity patterns affecting the data up to $0.99 r$, where $r$ is the solar disc radius. By this compensation we obtained contrast images. A slightly reduced disc area up to $0.98 r$ was used for the computation of the photometric sums in order to remove the effect of any possible artefacts very close to the limb.

Figure 1 shows examples of the intensity images analysed in our study, as well as the corresponding contrast images obtained from our processing. Also shown (bottom panels) are profiles along the horizontal and vertical cross-sections of the contrast images (marked by the dashed green lines in the contrast images). In particular, these profiles traverse facular and sunspots regions on the contrast image. The contrast of faculae in the RP continuum is clearly lower than in RP Ca II K observations. In the RP blue, the contrast is higher than in the RP red. In both RP continuum bands, the contrast in sunspots is considerably higher than in faculae. Sunspot contrast is also higher compared to that of faculae in SF Ca II K, though the difference is less pronounced than in the RP continuum data. In the RP and RPS Ca II K data, the facular contrast is higher than the sunspot one. The facular contrast in RP and RPS data is higher than in SF

\footnotetext{
${ }^{2}$ Available at http://www.csun.edu/SanFernandoObservatory/ sfosolar.html
} 

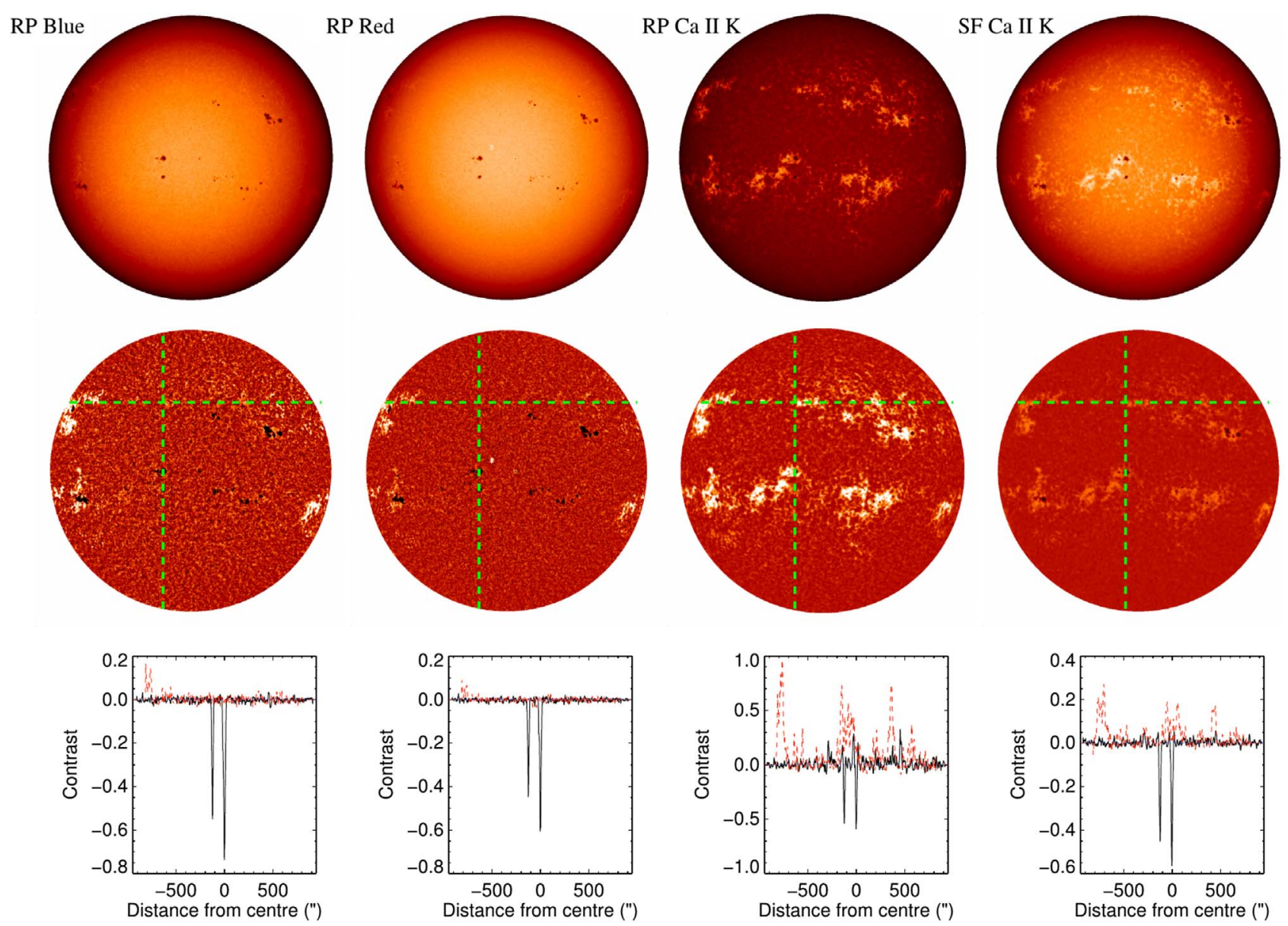

Fig. 1. Examples of observations analysed in this study from the Rome/PSPT (RP, columns 1-3) and SFO/CFDT2 (SF, column 4) telescopes. The images were taken on 31 March 2000 in the blue (1st column) and red (2nd column) continuum, and in the Ca II K line (3rd and 4th columns). The panels present raw (after the calibration of the CCD) intensity images (top row), limb-darkening-compensated contrast images (middle row), as well as horizontal (red) and vertical (black) profiles (bottom row) along the cross-sections of the contrast images shown in the middle row, as marked by the dashed green lines. The images have been rotated to display the solar north pole at the top. The raw images are shown to their entire range of values, while the contrast images are shown in the range $[-0.05,0.05]$ and $[-0.5,0.5]$ for the continuum and Ca II K images, respectively. In the contrast images, regions outside the ranges listed above are saturated and appear black or white.

Ca II $\mathrm{K}$ data. These relations between contrast values in the various data are consistent with the narrower bandwidth used for the RP and RPS observations compared to that employed for the SF data.

\subsection{TSI series}

In this study, we considered two original instrumental TSI measurement series, three TSI composites, and three model TSI reconstructions. The original instrumental records are the daily TSI measurements from the SORCE/TIM ${ }^{3}$ (version 18 , February 2020; Kopp \& Lawrence, 2005) and SOHO/VIRGO 4 (version 6.5, May 2018; Fröhlich et al., 1997) covering the time intervals from 2003 to 2020 and from 1996 to 2018, respectively. The considered TSI composites are the ACRIM ${ }^{5}$ (Active Cavity Radiometer Irradiance Monitor, which is the instrument taken as the reference by Willson, 1997), PMOD ${ }^{4}$ (named after

\footnotetext{
3 Available at the LISIRD archive at https://lasp.colorado.edu/lisird/

${ }^{4}$ Available at https://www.pmodwrc.ch

5 Available at http://www.acrim.com
}

Physikalisch-Meteorologisches Observatorium Davos; version 42.65; Fröhlich, 2006), and $\mathrm{RMIB}^{6}$ (named after Royal Meteorological Institute of Belgium, in french called IRMB; Dewitte et al., 2004; Dewitte \& Nevens, 2016), covering the periods 1978-2013, 1978-2018, and 1984-2020, respectively. These TSI composites are created by daisy-chaining, i.e. using the daily measurements of one instrument as the reference to calibrate the daily records of the second one, which then acts as the reference for the next one, etc. Finally, to compare the results of our study with others, we also considered the daily TSI reconstructions with the SATIRE-S ${ }^{7}$ (Spectral And Total Irradiance REconstruction; Yeo et al., 2014), EMPIRE ${ }^{7}$ (EMPirical Irradiance REconstruction; Yeo et al., 2017a), and NRLTSI ${ }^{8}$ (Naval Research Laboratory TSI; Lean, 2018) models.

All these models describe the irradiance variability through the varying contributions of sunspot darkening and facular

\footnotetext{
${ }_{6}^{6}$ Available at ftp://gerb.oma.be/steven/RMIB_TSI_composite/

${ }^{7}$ Available at http://www2.mps.mpg.de/projects/sun-climate/data. html

${ }^{8}$ Available at https://www.ncei.noaa.gov/data/total-solar-irradiance/
} 
brightening. SATIRE-S is a semi-empirical model. It employs the areas and positions on the solar disc of the various features at a given time as well as their time-invariant brightness contrasts as input. The brightness contrasts as a function of wavelength and position on the solar disc are obtained with the radiative transfer simulations from semi-empirical atmospheric models (Unruh et al., 1999). The disc area coverage by the surface features is derived from magnetograms and continuum filtergrams acquired from ground- and space-borne telescopes since 1974 (see e.g. Yeo et al., 2014, for more details). EMPIRE and NRLTSI are empirical models that linearly combine proxies of sunspot darkening and facular brightening to match the model outcome to the observed TSI changes. Whereas NRLTSI uses the ordinary least squares regression, EMPIRE uses the orthogonal distance regression accounting for the error in both variables. Both models use the $\mathrm{Mg}$ II index and sunspot areas as input over the period considered in this study.

\section{Variation of the photometric sums with the solar cycle}

Figure 2 shows the $\Sigma_{\mathrm{k}}, \Sigma_{\mathrm{b}}$, and $\Sigma_{\mathrm{r}}$ series obtained from the processing of the RP Ca II K, blue, and red images. All of them vary with the solar cycle, although with different amplitudes. In particular, the amplitude of the variation of $\Sigma_{b}$ and $\Sigma_{r}$ is considerably lower than that of $\Sigma_{\mathrm{k}}$, with the maximum absolute value of $\sim 1500$, which is an order of magnitude lower than that of $\Sigma_{\mathrm{k}}$.

The orange line shows the originally derived $\Sigma_{\mathrm{k}}, \Sigma_{\mathrm{b}}$, and $\Sigma_{\mathrm{r}}$. As was already suggested by Figure 1, the contribution of facular regions in $\mathrm{Ca}$ II $\mathrm{K}$ images is considerably higher than that of sunspots, leading to an in-phase variation of $\Sigma_{k}$ with the solar cycle, i.e. the Sun in the Ca II K line appears brighter during periods of activity maxima. In the red and blue continuum images, where the contrast of faculae is generally lower than that of sunspots, the balance is rather shaky and $\Sigma_{\mathrm{b}}$ and $\Sigma_{\mathrm{r}}$ might vary in anti-phase with the solar cycle, i.e. the Sun in the specific continuum intervals might appear marginally darker during periods of activity maxima. This is in agreement with result of Preminger et al. (2011). However, as discussed below and as also argued by Peck \& Rast (2015), this result turns to be extremely sensitive to the image processing, in particular, to the accuracy of the determination of the CLV.

In the following, we assess the potential uncertainties in the derived photometric sum indices. We use two different approaches to identify the QS level on the contrast images and to evaluate its variation with time. This allows us to test whether the processing had introduced any bias in the QS level. Here, we focus on the continuum data since such a test on Ca II K observations has already been performed by Chatzistergos (2017) and Chatzistergos et al. (2018b) using synthetic images.

Both methods make the underlying assumption that the distribution of the image contrast values is Gaussian, with active regions contributing to the wings of the distribution, but they take different approaches to identify the mean level of the QS regions. The first method was proposed by Nesme-Ribes et al. (1996) and was also used by Chatzistergos et al. (2019b, 2020a). An iterative process is used to estimate the level of the QS. Initially, the mean contrast, $\bar{C}$, and the standard

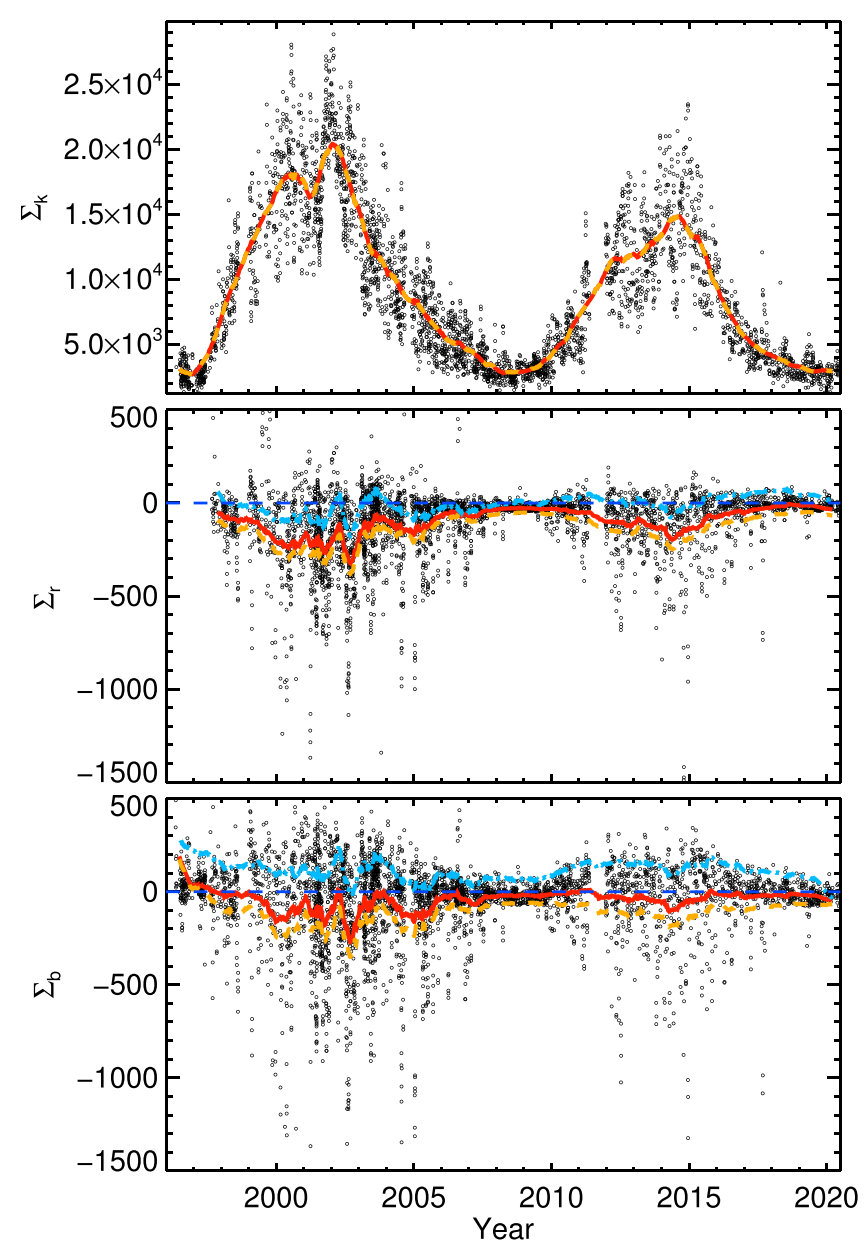

Fig. 2. Photometric sums from RP Ca II K (top), red (middle), and blue (bottom) observations. Daily values are shown in black, while 180-day running averages are shown in red. We note that the photometric sum indices were adjusted, following the method by Nesme-Ribes et al. (1996), to take into account the bias of the QS level (see Sect. 3). The dashed orange line shows the 180-day running average of the photometric sums prior to this adjustment, while the dash-dotted ciel line (only for the continuum data) was obtained following the approach by Preminger et al. (2002). The dashed blue horizontal line denotes the 0 -level of photometric sum.

deviation, $\sigma$, of the contrast values within the disc are computed. Then the mean and the standard deviation are re-calculated iteratively, each time leaving out regions with values outside the interval $\bar{C} \pm k \sigma$, where $k$ is a constant. The value of $k$ is taken between 0.1 and 3.0, and using a bisector approach, we search for the lowest value of the mean contrast among all $k$ values. This lowest mean contrast value is then adopted as the one representing the QS.

The second approach is an adaptation of the method used by Preminger et al. (2002) to analyse the potential bias in the SF data. For this, we compute the histogram of contrast values for each RP red and blue image, where the contrast values are grouped into bins of $10^{-5}$ width. A consistent image processing would return profiles centred at a value constant over the solar cycle. Following Preminger et al. (2002), we fit two Gaussians to the histogram of contrast values for each processed image, 

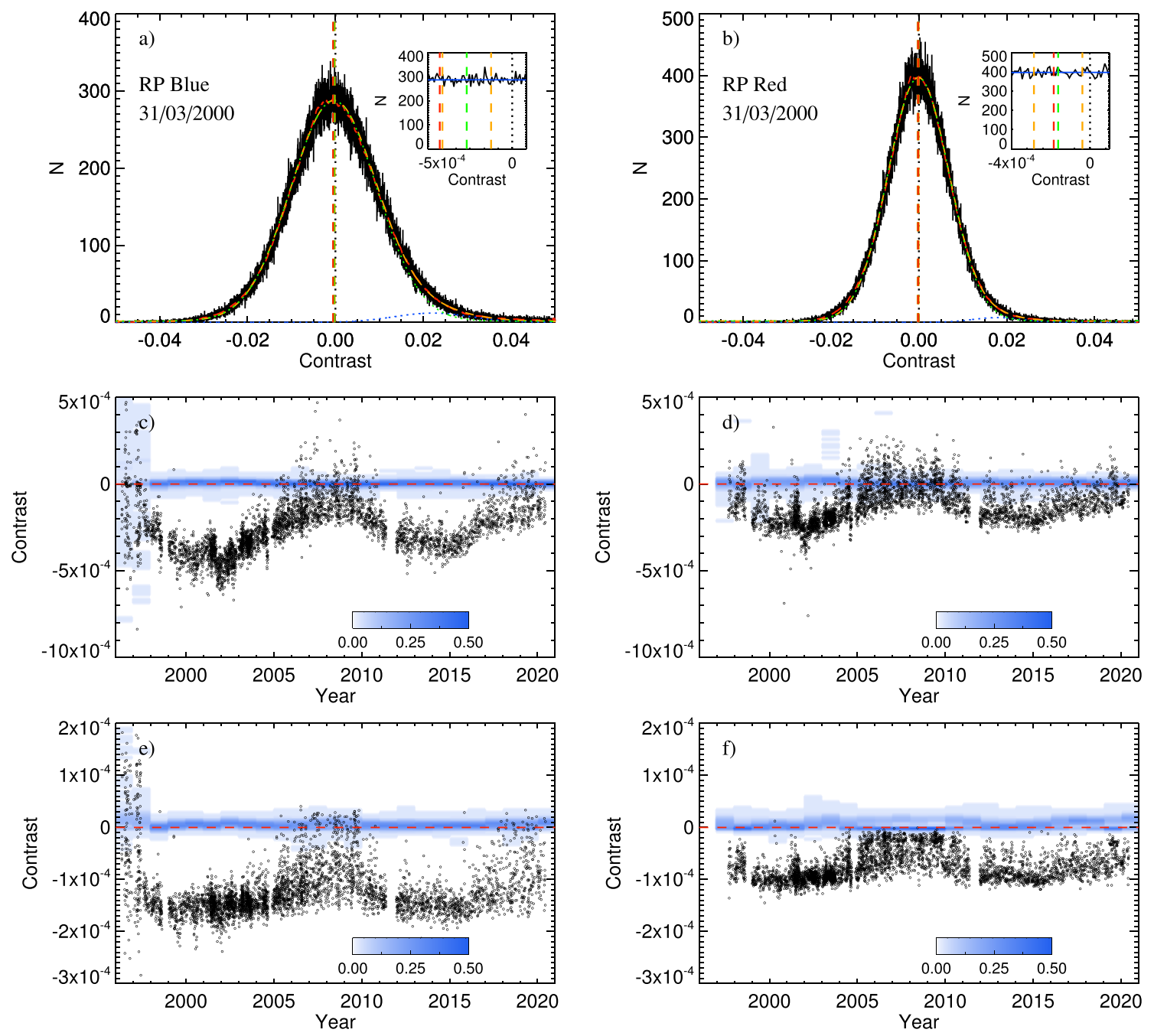

Fig. 3. Panels a and b: Histogram of pixel values (black) of the RP blue (left) and red (right) contrast images shown in the middle row of Figure 1. The fit of the sum of two Gaussians to the histogram of contrast values is shown in orange, while the individual Gaussian functions are shown in green and blue. Also shown in red is the 100-point running mean of the histogram. The insets show enlargements of the central parts to highlight the differences between the various quantities discussed in Section 3. In particular, the dashed vertical lines show the location of the central Gaussian function for this particular image (red) as well as the mean (green) and the $1 \sigma$ interval (orange) from all RP images, respectively. The black dotted vertical line denotes zero contrast. Panels c-f: Temporal variation of the QS level determined with the methods by Preminger et al. (2002, panels c and d) and by Nesme-Ribes et al. (1996, panels e and f) for all RP blue (left) and red (right) observations. The dashed red line marks the zero contrast level. Also shown are PDFs of the uncertainty of the QS bias estimations due to the pixel-by-pixel errors of the image processing as evaluated with a Monte-Carlo simulation (see Sect. 3 for more information). The PDFs are displayed in bins of 1 year and $10^{-5}$ in contrast and they are colour coded as indicated by the colour bars.

one for the central part, representing the QS regions, and one for the wing representing the bright regions. To confine the central part, we iteratively reduce the considered interval and fit a Gaussian function, searching for the contrast range for which the lowest $\chi^{2}$ of the Gaussian fit is achieved. The location of the peak of the central Gaussian is taken to represent the mean level of the QS regions. The residual between the distribution and the central Gaussian in the bright wing is then fit with another Gaussian function. Preminger et al. (2002) performed the fit on 7 annuli equally spaced in $\mu$ rather than the whole disc. They also did not iterate. Despite this modification, we will still refer to this approach as the one by Preminger et al. (2002).
As an example, Figure 3 shows the histograms of the RP red and blue images presented in Figure 1 along with the evolution of the QS level computed with the two approaches mentioned above for all continuum images analysed in this study. Both approaches indicate a weak although non-negligible variation of the QS level in anti-phase with the solar cycle. The QS level derived with the method of Preminger et al. (2002) varies by more than when derived with the method of Nesme-Ribes et al. (1996). The mean and the standard deviation of the QS level obtained with the method of Preminger et al. (2002) are $-1 \pm 4.9 \times 10^{-4}$ and $-2.5 \pm 3.8 \times 10^{-4}$ for the RP red and blue images, respectively. The mean and the standard deviation of the QS level determined with the method of Nesme-Ribes 
et al. (1996) are $-0.7 \pm 0.3 \times 10-4$ and $-1.2 \pm 6.5 \times 10^{-4}$ for the RP red and blue images, respectively. These findings suggest that the QS level in the RP red and blue images calculated with both approaches is affected by the solar activity. In particular, the QS level is underestimated during activity maxima. Around activity minima, both methods return relatively accurate results. The variation is stronger in the RP blue than in the RP red images. We found no dependence of the bias on the heliocentric angle. However, when applied on narrow annuli, the results are less accurate for both methods. The reason is the significantly reduced statistics compared to the case when the entire disc is considered. It is worth noting that the results obtained with the method by Nesme-Ribes et al. (1996) are qualitatively similar to those obtained by Chatzistergos (2017) from the analysis of Ca II K data, for which we found a bias of $\sim 1 \pm 1 \times 10^{-4}$ with a minute anti-phase relation to the solar cycle.

In Chatzistergos (2017), we showed that the accuracy of recovering the pixel-by-pixel contrast values in synthetic $\mathrm{Ca}$ II K images was on average $10^{-3}$ (the RMS error reached up to $3 \times 10^{-3}$ ). We also showed that our approach worked better than other published methods. This pixel-by-pixel error is one order of magnitude higher than the QS bias reported here with both methods. These processing errors can affect the determination of the QS level. To estimate the sensitivity of the applied methods on the pixel-by-pixel errors of our processing, we performed a Monte Carlo simulation. In particular, we used all RP contrast images and imposed a random offset on contrast values in each pixel. We then recomputed the QS level with the two considered methods. We have done this for 100 different sets of uniformly distributed random contrast offsets within the range -3 to $3 \times 10^{-3}$. The distribution of the differences between the QS level computed from the offset and from the original images gives us an estimate of the uncertainty of the QS bias estimation due to processing errors. These distributions for annual bins are shown in Figure 3. We find the uncertainty due to the pixel-by-pixel errors to typically lie below $10^{-4}$ and $3 \times 10^{-5}$ for the methods by Preminger et al. (2002) and Nesme-Ribes et al. (1996), respectively. This suggests that the estimation of the QS bias is not strongly affected by the pixel-by-pixel processing errors of our method. The uncertainty with both methods is comparable, although the effect is slightly stronger when using the method by Preminger et al. (2002). The derived QS bias for RP blue data is higher over the years 1996 and 1997, thus rendering our results over these two years rather uncertain. We note, however, that this uncertainty estimate of the QS bias is only valid for images processed with our method and it is expected to be higher when using other published processing methods.

The results presented in Figure 3 suggest that to accurately determine the photometric sums $\Sigma_{\mathrm{b}}$ and $\Sigma_{\mathrm{r}}$, we need to account for the bias in the level of the QS definition. Therefore, we repeated the computation of the $\Sigma$ series after the appropriate adjustment of the QS level in each image, using both the methods by Preminger et al. (2002) and Nesme-Ribes et al. (1996). These adjusted photometric sum series are shown in Figure 2. The RMS difference between the $\Sigma$ series adjusted with the method by Nesme-Ribes et al. (1996) and the original $\Sigma$ series is 115 for both the red and the blue data. Using the method by Preminger et al. (2002) the RMS differences are 294 and 384 for the red and blue observations, respectively. For comparison, the corresponding RMS difference for the Ca II K data when using the method by Nesme-Ribes et al. (1996) is 146. The adjustment to account for the bias of the $\Sigma_{\mathrm{k}}$ series has barely any effect on the series since the adjustment is two orders of magnitude lower than the $\Sigma$ values. However, for the blue and red observations, the correction is comparable to the $\Sigma$ values.

After the correction, the temporal profile of $\Sigma_{b}$ and $\Sigma_{\mathrm{r}}$ series changes both in terms of the amplitude and the shape (see Fig. 2). In particular, with the QS level corrected with the method by Nesme-Ribes et al. (1996, red curves) the profile of $\Sigma_{\mathrm{b}}$ becomes essentially flat, with only a tiny decrease over the maximum of solar cycle 24 , whereas the variation of the $\Sigma_{\mathrm{r}}$ remains in anti-phase with the solar cycle. After the correction of the QS level with the method by Preminger et al. (2002) the variation of $\Sigma_{b}$ turns to be in-phase with the solar cycle, while the profile of $\Sigma_{\mathrm{r}}$ becomes essentially flat. However, while the adjusted $\Sigma_{\mathrm{r}}$ and $\Sigma_{\mathrm{b}}$ are, on average, close to zero at minima when the method by Nesme-Ribes et al. (1996) is used, the corresponding values for $\Sigma_{\mathrm{b}}$ with the corrections following Preminger et al. (2002) are roughly around 100. This points to a potential over-correction with the method by Preminger et al. (2002). We thus conclude, that the uncertainty in the definition of the QS bias, independently of the method used, as well as the potentially remaining dependence of the QS bias on solar activity render the phase of the variation of $\Sigma_{\mathrm{r}}$ and $\Sigma_{\mathrm{b}}$ significantly uncertain. We consider the method by NesmeRibes et al. (1996) to return more accurate results than the method by Preminger et al. (2002) as applied here. In the following, we will use the $\Sigma$ series adjusted for the QS bias with the method by Nesme-Ribes et al. (1996) being the intermediate case found here.

We note that Preminger et al. (2002) also reported a variation of $\Sigma_{\mathrm{r}}$ derived from SF red continuum images similar to what we obtain here. However, they argued that the offset was too small to have any effect on the solar-cycle phase of the $\Sigma_{\mathrm{r}}$ variability. Specifically, they found a mean bias in the QS level during an activity maximum of only $\sim 5 \times 10^{-4}$, although this offset doubled towards the limb. They argued that this bias introduced a mean offset in $\Sigma_{\mathrm{r}}$ of 120 and -13 for 1989 and 1996, corresponding to activity maximum and minimum periods, respectively. These values are consistent in amplitude with the ones derived here.

Puiu (2019) analysed the accuracy of $\Sigma$ evaluation by processing a small sub-sample of RPS observations with methods different than those employed in this study (see Sect. 5 for a brief description of their method). In particular, they studied the effect of various parameters of the processing on their results and especially on the photometric sums. Their final adopted parameters returned contrast images favouring the variation of $\Sigma_{\mathrm{r}}$ in anti-phase with the solar cycle. However, their analysis showed that generally the image processing can result in a reversal of the phase of the relation between $\Sigma_{\mathrm{r}}$ and the solar cycle.

\section{Long-term trends in reconstructed TSI}

Using the computed photometric sums and equation (1), we now reconstruct the TSI. The free parameters of the multiple linear regression model are determined by fitting various 

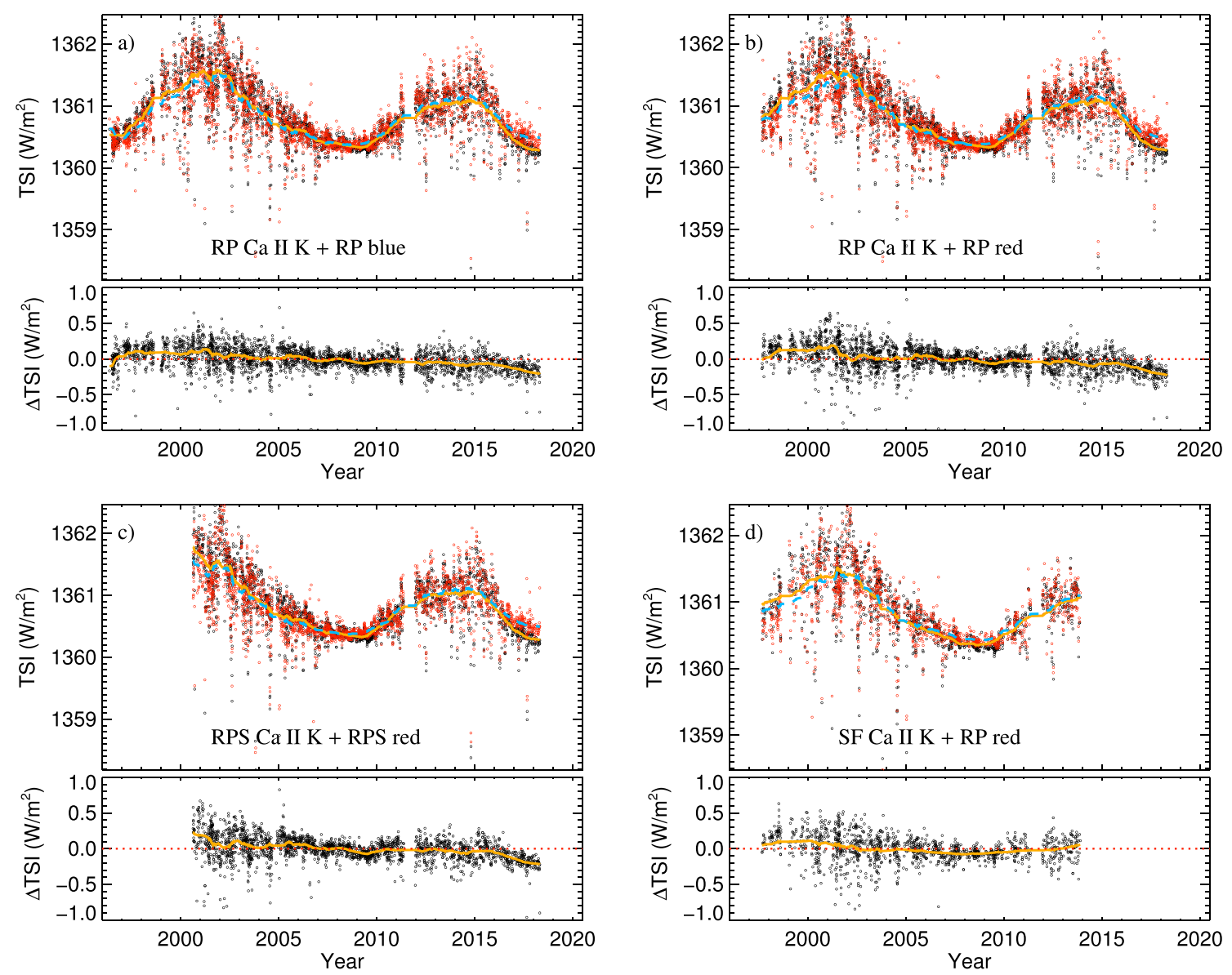

Fig. 4. Comparison between the reconstructed TSI series (red circles for daily values and dashed ciel line for the 180-day running mean in the top part of each panel) and the PMOD TSI composite series (black circles for daily values and solid orange line for 180-day running mean values). At the bottom of each panel, the corresponding difference between the PMOD TSI and the reconstructed TSI (i.e. PMOD minus our reconstruction) is shown (black circles for daily values and orange line for the 180-day running mean). TSI series are reconstructed using the following data: RP Ca II K and RP blue (a); RP Ca II K and RP red (b); RPS Ca II K and RPS red (c); and SF Ca II K and RP red (d) observations. For all reconstructions, the PMOD TSI composite was used as the reference when deriving the regression coefficients.

combinations of the photometric sums, $\Sigma$, to the PMOD TSI composite. We consider the $\Sigma$ series derived from the following five pairs of observations: RP Ca II K and RP red, RP Ca II K and RP blue, RPS Ca II K and RPS red, as well as SF Ca II K and RP red and SF Ca II K and RP blue. Figure 4 shows the reconstructed TSI along with the PMOD TSI composite. Table 2 summarises the parameters of the fit, along with the RMS difference, and linear correlation coefficient, $R$, for each reconstruction. We found that the best TSI reconstruction is achieved with RP Ca II K and RP blue observations, with a RMS difference and $R$ to the PMOD TSI series of $0.15 \mathrm{Wm}^{-2}$ and 0.96 , respectively. The TSI reconstructions from RP red observations exhibit a slightly higher scatter than those derived from RP blue data. The quality of the TSI reconstructions is similar to the previous combinations when RPS or SF Ca II K data are used. The PMOD TSI shows a somewhat stronger declining trend than the reconstruction. This is discussed in more detail below.

The empirical TSI reconstructions, based on the regression of solar activity indices to the TSI measurements, are obviously subject to the uncertainties in those measurements. Therefore, we have done various TSI reconstructions by varying the TSI reference series used for the regression. Table 2 lists the parameters of the fit, along with the RMS difference, and $R$ for each reconstructions, whereas Figure 5 shows the residual between each considered TSI reference record and the corresponding reconstruction from RP Ca II K and RP blue observations.

We generally found a good agreement between our TSI reconstructions and the various published TSI series (see Sect. 2.3). Most reconstructions show the same characteristics. In particular, as in the case of PMOD, a slightly declining trend is seen in the residuals with time. Exceptions are the reconstructions that use the SORCE/TIM data as reference, which display a slightly increasing trend of $1.3 \times 10^{-2} \mathrm{Wm}^{-2} \mathrm{y}^{-1}$. This might partly be because the SORCE/TIM data do not cover the maximum of cycle 23 . However, we note that Dewitte \& Nevens (2016) and Woods et al. (2018) reported an increasing drift of $3.43 \times 10^{-2} \mathrm{Wm}^{-2} \mathrm{y}^{-1}$ in the TSI values from SORCE/TIM over the same period. We also found that our TSI reconstruction with the ACRIM composite as the reference displays the strongest trend of residuals to the measurements. This is most likely due to issues with the ACRIM composite series. We also computed the $\mathrm{R}$ and RMS differences between our reconstructed 
Table 2. Results of reconstructing TSI.

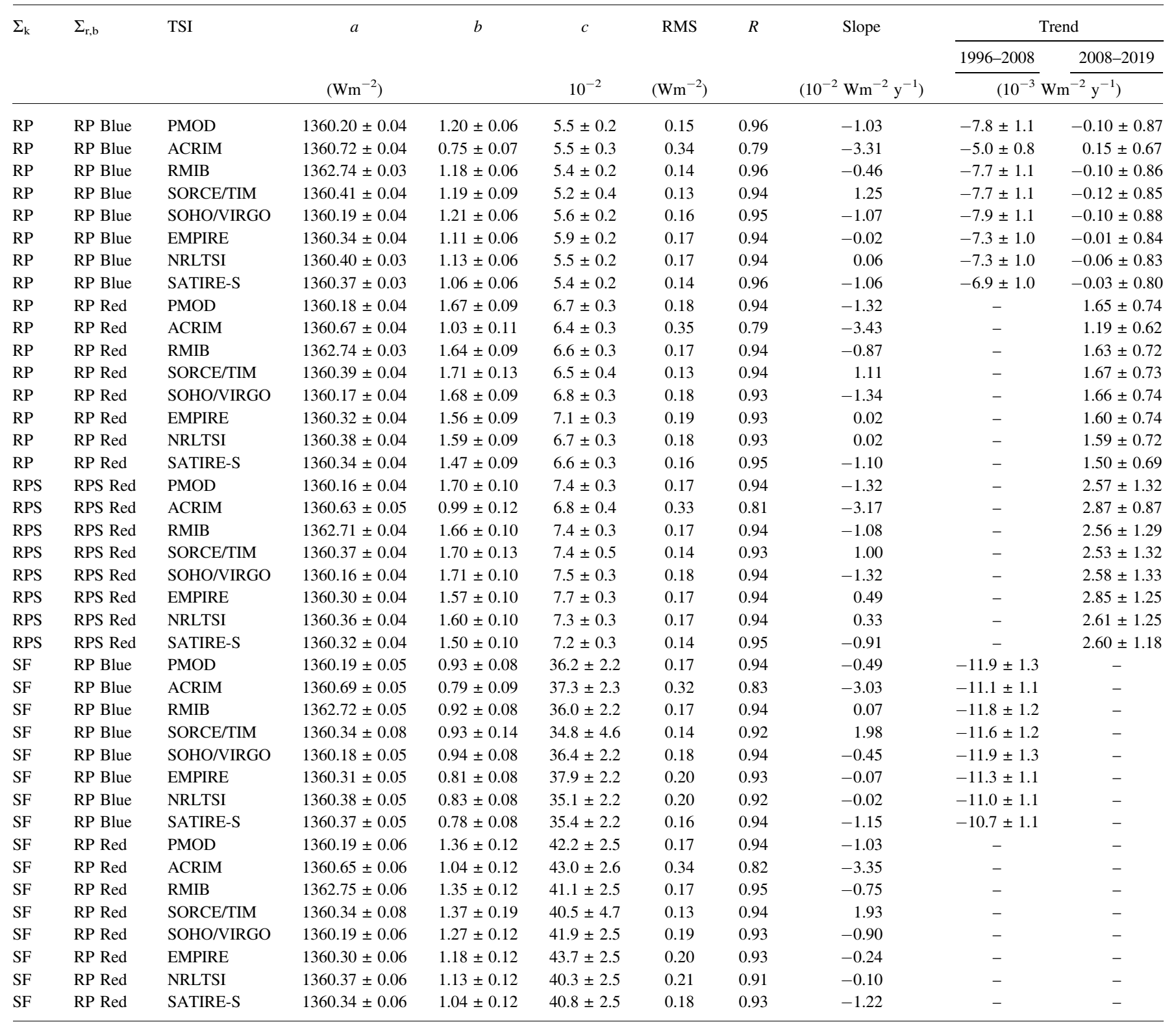

Note. Columns are: Ca II K and continuum observations employed to compute $\Sigma_{\mathrm{k}}, \Sigma_{\mathrm{b}}$, and $\Sigma_{\mathrm{r}}$, TSI reference series used for the regression, best fit parameters of equation (1), RMS difference, linear correlation coefficient $R$, and slope of residual between the reconstructed TSI series and the reference one, and long-term trend as defined with a linear fit during activity minimum periods.

TSI and the various reference series after detrending our series. For detrending we used the residual trend listed in Table 2. We find the RMS differences to generally decrease slightly in all cases, however qualitatively we report the same findings.

For a more quantitative comparison, we have performed a linear fit to the reconstructed TSI over each two subsequent solar activity minima (i.e. 1996 vs. 2008 and 2008 vs. 2019). For this, we used 12-month intervals centered at the minimum of each of the 3 cycles. The dates of the minima were taken as 01 August 1996, 01 December 2008, and 01 October 2019 (Hathaway, 2015). We note that at the time of writing this manuscript there is no official starting date for solar cycle 25 and hence the value used here might change somewhat, although probably not much (see e.g. Alterman et al., 2020). Therefore, we use the last year for which the data are currently available for the last minimum.

Figure 6 shows the reconstructions for the various TSI measurement records employed as the reference, along with the linear fits to the TSI values during the activity minima. For ease of comparison, we offset the reconstructed series so to match their mean values over a 12-month interval centred at the minimum of 2008 to that of the PMOD TSI series. While the various reconstructions differ slightly in the magnitude of the solar cycle variation, they agree remarkably well during activity minima, 


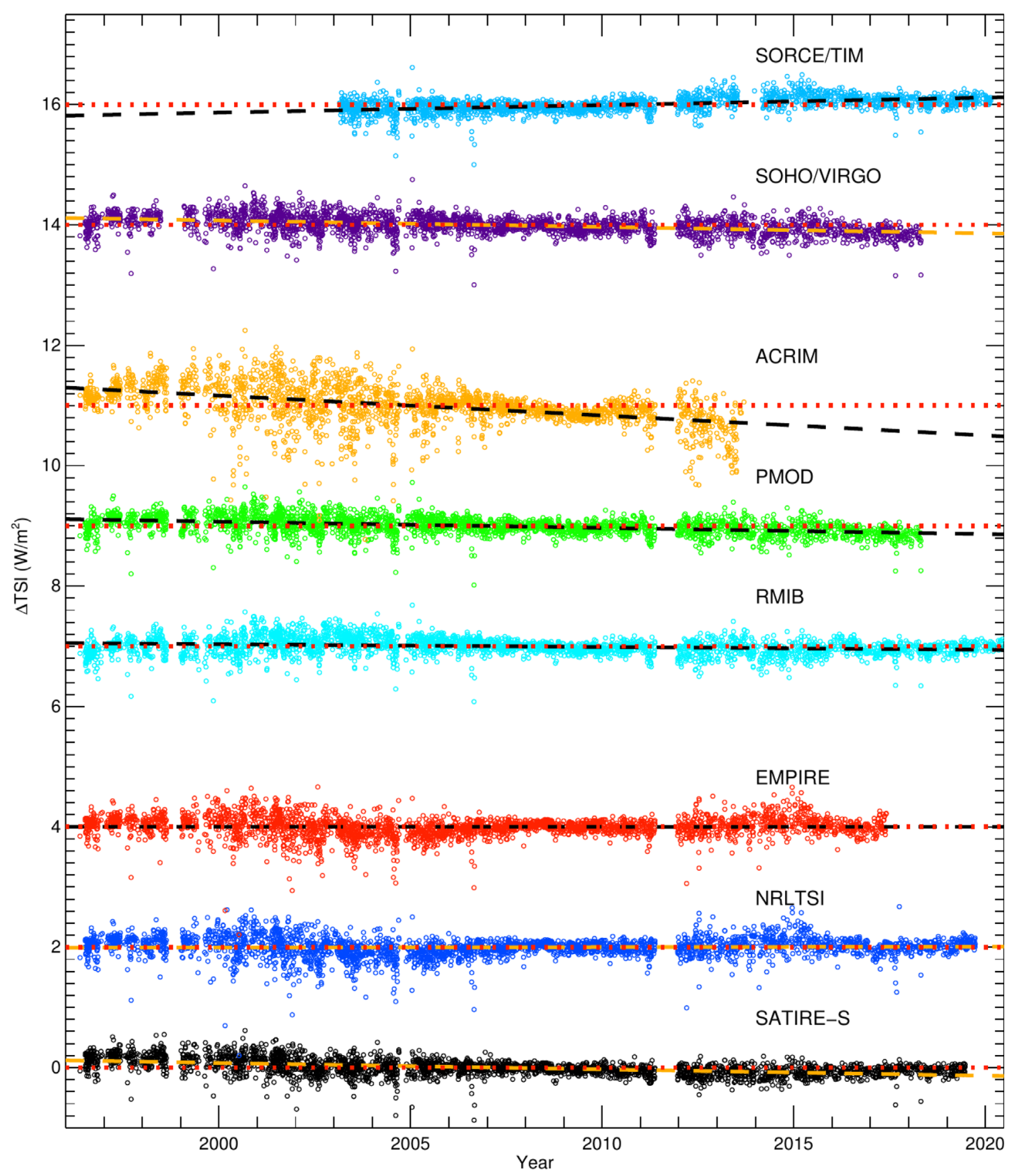

Fig. 5. Differences between the various reference TSI datasets (see the legend) and the corresponding TSI reconstructions from RP Ca II K and blue observations. The residuals have been offset to improve visibility. The dotted red lines denote differences of 0 , while the dashed lines are linear fits to the residuals.

showing essentially the same minimum-to-minimum changes. Thus, the choice of the reference TSI dataset is not decisive for the long-term trend in the reconstructed record. We also note that the long-term trends of the TSI series derived from the PMOD and RMIB TSI composite data employed as the references are remarkably close to each other, with differences being less than $2.1 \times 10^{-4} \mathrm{Wm}^{-2} \mathrm{y}^{-1}$. However, this is mainly due to the similarity of the PMOD and RMIB TSI composites over the selected periods.
The TSI reconstruction based on $\mathrm{RP} \mathrm{Ca}$ II $\mathrm{K}$ and $\mathrm{RP}$ blue images that uses the PMOD data as the reference shows a long-term trend of $-7.8_{-0.8}^{+4.9} \times 10^{-3}$ and $-0.1_{-0.02}^{+0.25} \times$ $10^{-3} \mathrm{Wm}^{-2} \mathrm{y}^{-1}$ between the minima 1996/2008 and 2008/ 2019, respectively, where the uncertainty ranges account for the spread in results derived using all other published TSI data as the reference. The uncertainty of the fit is roughly $10^{-3} \mathrm{Wm}^{-2} \mathrm{y}^{-1}$ and is lower than the uncertainty due to using different TSI reference series. The declining trend over the 

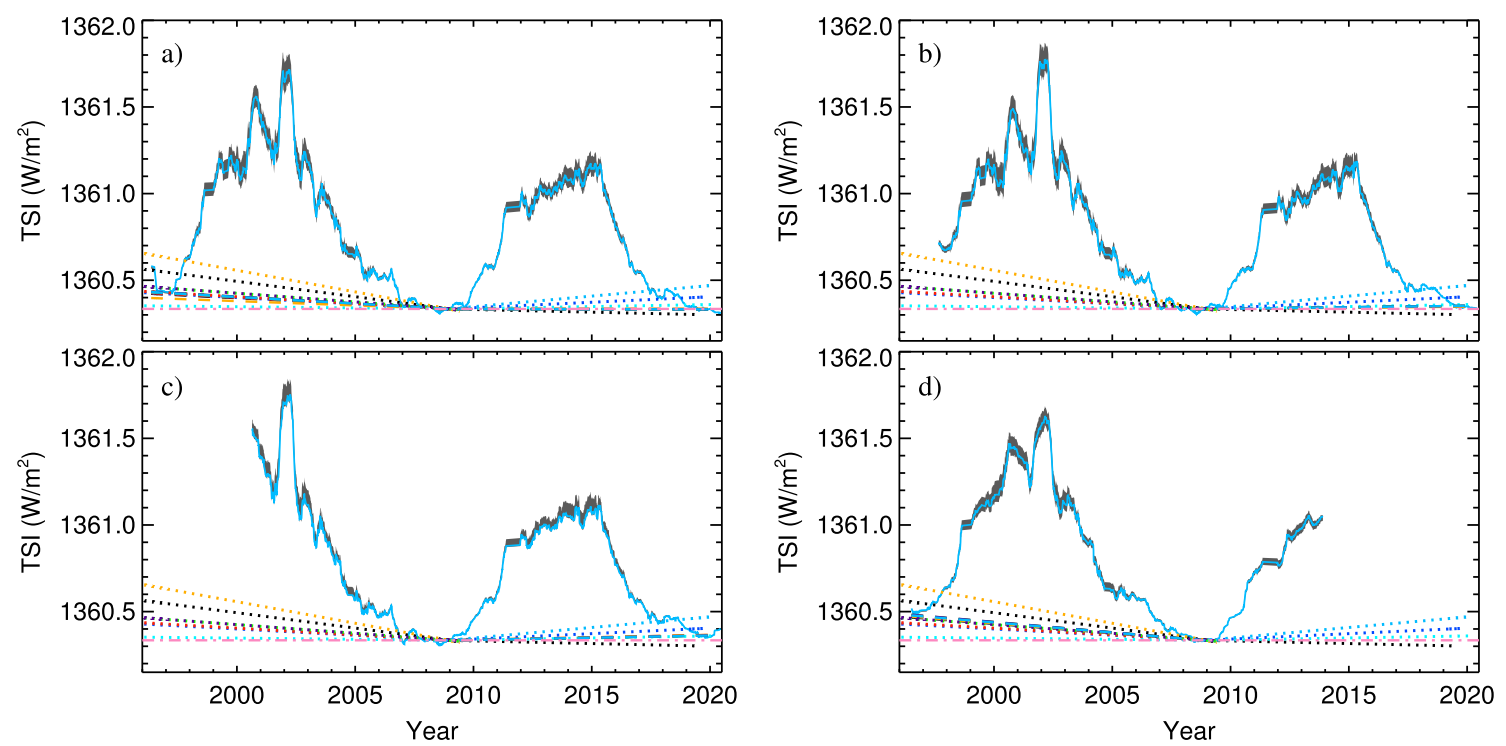

Fig. 6. TSI reconstructed with various reference series using the following images: RP Ca II K and RP blue (a); RP Ca II K and RP red (b); RPS CA II K and RPS red (c); and SF Ca II K and RP blue (d). Colours are the same as in Figure 5. The solid line is 81-day running mean values for the series with PMOD as the reference, while the grey shaded surface shows the range covered by the reconstructions with all other TSI reference series. The dashed and dotted lines are linear fits to the reconstructed and reference TSI values, respectively, during subsequent activity minima. All series were offset to match their mean value within a 12-month interval centered at the minimum of 2008 to that of the PMOD TSI composite. The value of the PMOD TSI composite over this period is marked with the horizontal dash-dotted pink line. The various dashed and dotted lines extend only to the intervals for which their respective data cover the complete two surrounding minima.

1996/2008 minima is statistically significant based on a Student's $t$-test, but this is not the case for the declining trend over the 2008/2019 minima. However, using different input data returns slightly different values for the long-term trend. For example, for the change between 1996 and 2008 we found $-11.9_{-0.06}^{+1.2} \times 10^{-3} \mathrm{Wm}^{-2} \mathrm{y}^{-1}$ when using SF Ca II K and RP blue observations, which is slightly higher than obtained from RP Ca II K and blue data. Between the 2008 and 2019 minima, the trend marginally changes sign and is $2.6_{-0.1}^{+0.3} \times$ $10^{-3} \mathrm{Wm}^{-2} \mathrm{y}^{-1}$ with RPS Ca II K and red observations, while it becomes $1.6_{-0.1}^{+0.7} \times 10^{-3} \mathrm{Wm}^{-2} \mathrm{y}^{-1}$ with RP Ca II K and red observations. Overall, our reconstructions suggest a weak decline in TSI from 1996 to 2008, and essentially no trend from 2008 to 2019.

Finally, for completeness, we have also compared our reconstructions to the widely used models, namely SATIRE-S, NRLTSI, and EMPIRE (see Sect. 2.3 for the description of the datasets and Table 2 and Figs. 5 and 6 for the results). Thereby, for an unbiased comparison, we also used the corresponding series as the reference. The lowest RMS difference and highest $R$ are obtained in comparison to SATIRE-S and EMPIRE reconstructions. The slope of the residual is somewhat closer to zero when the two empirical reconstructions, EMPIRE and NRLTSI, are used as reference. These models are similar in concept to ours, i.e. they are also based on linear regressions of the solar activity indices to the measured TSI, except that they use disc-integrated proxies. The slope of the residual between our reconstruction and SATIRE-S is closer to that obtained for most reconstructions using observational records as the reference (except SORCE/TIM). All differences are, however, rather small.

\section{Discussion}

In Section 3, we analysed the sensitivity of the $\Sigma$ series to the computation of the QS level in the images when processed with our method. We now consider also the effect of the QS computation on the $\Sigma$ series and the TSI reconstruction when using different processing methods. In particular, we consider the methods presented by Brandt \& Steinegger (1998), Walton et al. (1998), Worden et al. (1998), and Puiu (2019). All of these studies used full-disc Ca II K observations to reconstruct TSI.

Briefly, Brandt \& Steinegger (1998) and Walton et al. (1998) derive the QS CLV by considering image pixels grouped into concentric annuli, with the value of the QS within each annulus determined with a threshold in the cumulative histogram of intensity values and the median of intensity values, respectively. Since both of those methods assume that the CLV is perfectly radially symmetric, they are unable to account for some image artefacts that often affect the full-disc observations, such as the linear gradient of intensity values caused by the atmospheric extinction in the RP and RPS data. To account for this, we have added an extra step to perform a 2D linear fit to the contrast images resulting from the processing by Brandt \& Steinegger (1998) and Walton et al. (1998). This fit is then subtracted from the contrast images. The processing by Worden et al. (1998) incorporates 5th degree polynomial fits along lines in the vertical and horizontal directions, as well as in segments with the orientation of $45^{\circ}$ and $135^{\circ}$. Finally, Puiu (2019) calculates the background as Walton et al. (1998), but regions within and outside $0.7 r$ are processed separately. Additionally, active regions are excluded from the QS 

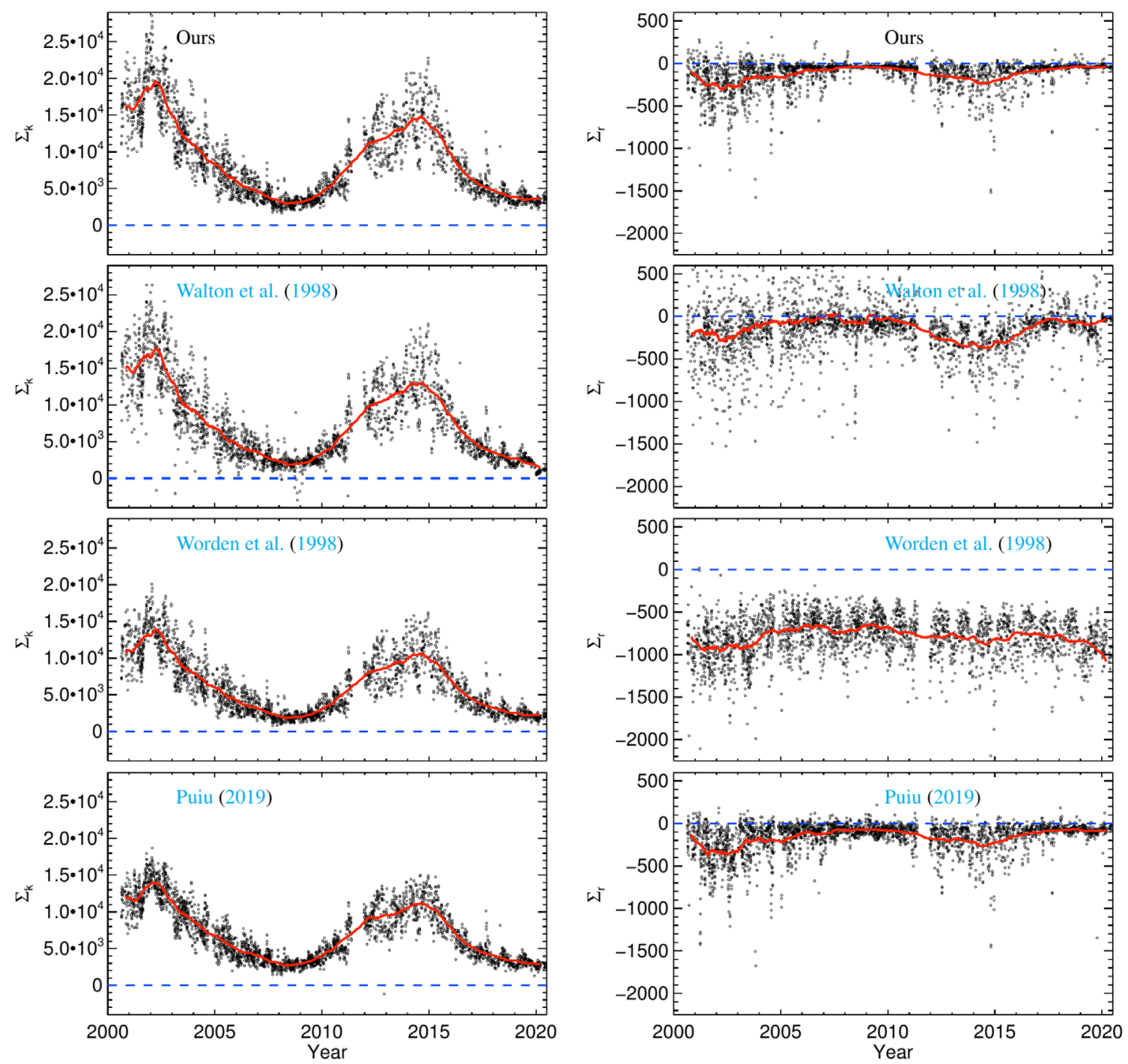

Fig. 7. Series of photometric sum indices derived from RPS Ca II K (left) and RPS red continuum (right) observations which were processed with our method (1st row) and the methods by Walton et al. (1998, 2nd row), Worden et al. (1998, 3rd row), and Puiu (2019, 4th row). Daily values are shown in black, while 180-day running averages are shown in red. The dashed blue horizontal line denotes the 0-level of photometric sum.

computation with an iterative procedure that follows the one proposed by Chatzistergos et al. (2018b) and used in this study. As the last steps, Puiu (2019) applies a linear vertical fit and a running window median filter to account for any residual intensity gradient in RPS data.

In Chatzistergos (2017) and Chatzistergos et al. (2018b), we studied the accuracy of the methods proposed by Brandt \& Steinegger (1998), Walton et al. (1998), and Worden et al. (1998) to remove the limb-darkening on synthetic Ca II K data. We showed that these methods performed worse than the ones employed in this study, by introducing artefacts that are subject to the solar cycle due to an inaccurate exclusion of active regions when defining the QS CLV. In particular, when using the methods by Brandt \& Steinegger (1998), Walton et al. (1998), and Worden et al. (1998), mean offsets for the QS level were up to 2,1 , and 2 orders of magnitude higher than the one obtained with the method employed here, respectively.
Furthermore, Puiu (2019) compared the accuracy of their processing to ours and also found the latter to be more accurate.

It is important to note that these error estimates were limited to $\mathrm{Ca}$ II $\mathrm{K}$ images, for which a significant source of errors is inaccurate accounting for active regions. On continuum images, where the disc coverage by active regions is significantly lower than in Ca II K images, other methods might fare better. Therefore, to check this, we used all these four methods to process the RPS Ca II K and red images, produce photometric sum series and reconstruct the TSI. We restricted this test to the RPS data for consistency with the analysis by Puiu (2019). Figure 7 (left panels) shows the $\Sigma_{\mathrm{k}}$ series derived from RPS Ca II K observations processed with our and three of the earlier methods. We do not show the results with the method by Brandt \& Steinegger (1998), because they are very similar to those by Walton et al. (1998). The $\Sigma_{\mathrm{k}}$ series obtained from our method exhibits the highest variability over the solar cycle. This suggests a potential 
T. Chatzistergos et al.: J. Space Weather Space Clim. 2020, 10, 45

Table 3. Results of reconstructing TSI over 2000-2020 from RPS contrast images processed with different methods.

\begin{tabular}{|c|c|c|c|c|c|c|c|c|c|}
\hline \multirow[t]{2}{*}{ Method } & \multicolumn{2}{|c|}{$R_{0}$} & \multirow{2}{*}{$\begin{array}{c}a \\
\left(\mathrm{Wm}^{-2}\right)\end{array}$} & \multirow[t]{2}{*}{$b$} & \multirow{2}{*}{$\begin{array}{c}c \\
10^{-2} \\
\end{array}$} & \multirow{2}{*}{$\begin{array}{c}\mathrm{RMS} \\
\left(\mathrm{Wm}^{-2}\right)\end{array}$} & \multirow[t]{2}{*}{$R$} & \multirow{2}{*}{$\begin{array}{c}\text { Slope } \\
\left(10^{-2} \mathrm{Wm}^{-2} \mathrm{y}^{-1}\right)\end{array}$} & \multirow{2}{*}{$\begin{array}{c}\text { Trend } \\
\left(10^{-3} \mathrm{Wm}^{-2} \mathrm{y}^{-1}\right)\end{array}$} \\
\hline & $\Sigma_{\mathrm{k}}$ & $\Sigma_{\mathrm{r}}$ & & & & & & & \\
\hline Ours & - & - & $1360.16 \pm 0.04$ & $1.70 \pm 0.10$ & $7.4 \pm 0.3$ & 0.17 & 0.94 & -1.32 & 2.57 \\
\hline Walton et al. (1998) & 0.98 & 0.21 & $1360.32 \pm 0.04$ & $0.23 \pm 0.05$ & $4.8 \pm 0.3$ & 0.37 & 0.68 & -1.29 & 1.3 \\
\hline Worden et al. (1998) & 0.99 & 0.51 & $1360.77 \pm 0.07$ & $0.58 \pm 0.07$ & $7.1 \pm 0.4$ & 0.35 & 0.72 & -1.59 & -10.4 \\
\hline Puiu (2019) & 0.97 & 0.88 & $1360.05 \pm 0.04$ & $1.33 \pm 0.09$ & $10.6 \pm 0.4$ & 0.23 & 0.89 & -1.79 & -1.5 \\
\hline
\end{tabular}

Notes. Columns are: processing technique applied on the RPS Ca II K and RPS red continuum data to compensate the CLV, linear correlation coefficient $R_{0}$ between the $\Sigma_{\mathrm{k}}$ and $\Sigma_{\mathrm{r}}$ series computed with our processing and those from the various tested methods, the best fit parameters of equation (1), RMS difference, linear correlation coefficient $R$, and slope of residual between the reconstructed TSI series and the PMOD TSI composite, and long-term trend as defined with a linear fit during the activity minimum period of 2008-2019.
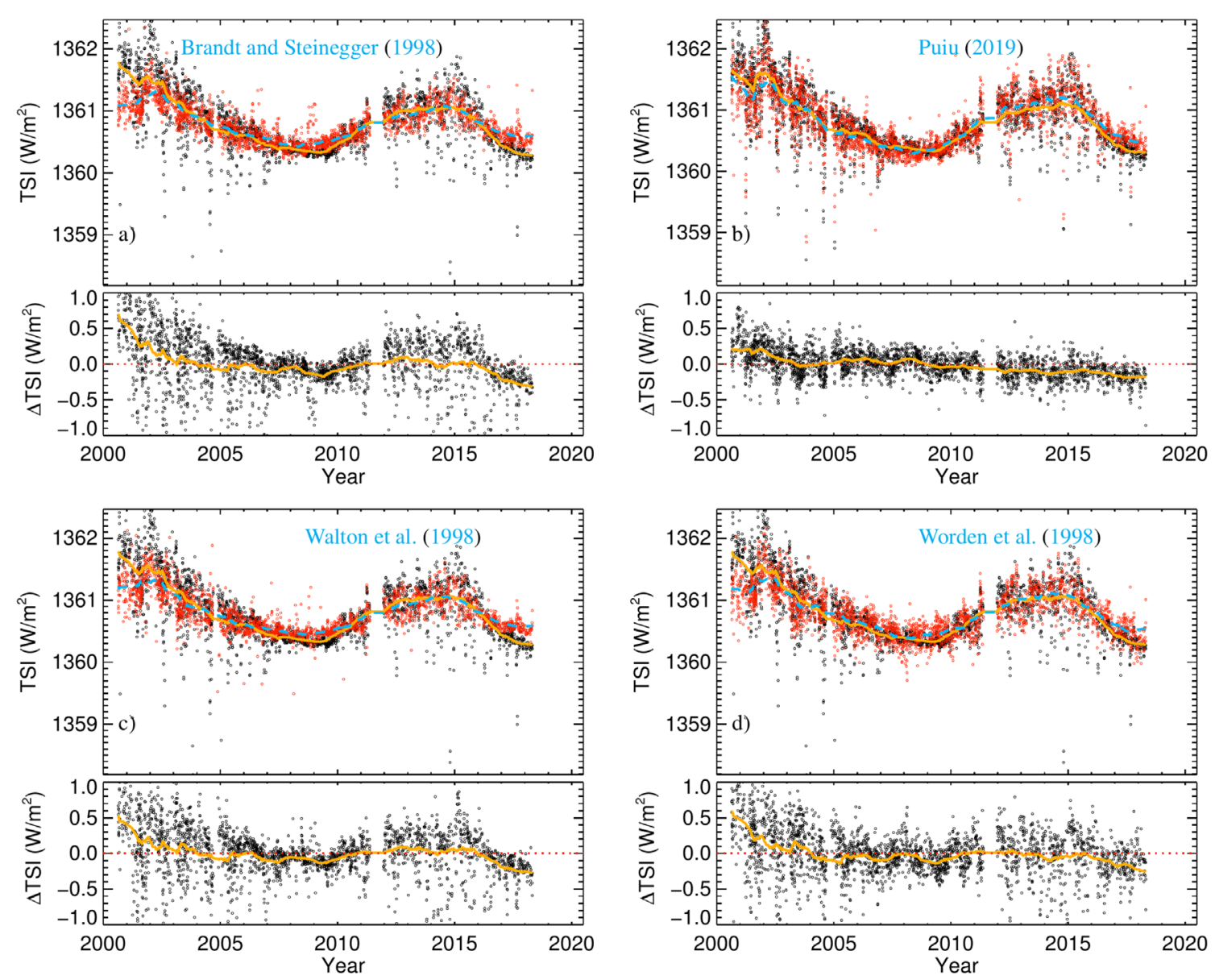

Fig. 8. Comparison between the PMOD TSI composite series (black circles for daily values and solid orange line for 180-day running mean values) and TSI series reconstructed (red circles for daily values and dashed ciel line for 180-day running mean values) from RPS Ca II K and RPS red observations processed with the methods by Brandt \& Steinegger (1998, panel a), Puiu (2019, panel b), Walton et al. (1998, panel c), and Worden et al. (1998, panel d). The PMOD TSI composite was taken as reference for all those reconstructions. Find more details in Section 5. At the bottom of each panel, the corresponding difference between the PMOD TSI and the reconstructed TSI is shown (black circles for daily values and orange line for the 180-day running mean).

suppression of the contrast of the bright regions by the other processing techniques, which is in agreement with the finding that these methods do not accurately exclude active regions when computing the QS CLV (Chatzistergos et al., 2018b).
Figure 7 (right panels) shows the $\Sigma_{\mathrm{r}}$ series derived from RPS red images by using our and three of the tested methods. All series exhibit a variation in anti-phase with the solar cycle. However, the series obtained with the methods by Brandt \& Steinegger (1998) and Walton et al. (1998) show high scatter 
and include many extreme values that are likely due to image artefacts unaccounted for by the image processing. For this reason, we ignored 77 images for which the processing by the method of Walton et al. (1998) produced $\Sigma_{\mathrm{r}}$ values $>2000$. We note that these results were obtained by applying the $2 \mathrm{D}$ linear fit, which might not accurately remove the linear gradient in the data. However, the results without the additional 2D fit were considerably worse, lacking any clear cycle variation. We also note that the 5 th degree polynomial used for the fitting along linear segments in the method by Worden et al. (1998) fails to reproduce the CLV in RPS red observations. This is in agreement with the findings by Puiu (2019). We found that the method by Puiu (2019) results in a distinct anti-phase relation between the $\Sigma_{\mathrm{r}}$ series and the solar cycle. The $\Sigma_{\mathrm{r}}$ series computed with the method by Puiu (2019) shows the best agreement among the various tested methods to that derived with our method, exhibiting a high correlation coefficient of 0.88 (see Table 3 ). This suggests that the method by Puiu (2019) performs better than the methods by Brandt \& Steinegger (1998), Walton et al. (1998), and Worden et al. (1998). The spread of $\Sigma_{\mathrm{r}}$ values during minima is greater when using the method by Puiu (2019) than with our method.

Table 3 summarizes the fit parameters (Eq. (1)) when using the $\Sigma$ series derived from all tested methods. Also listed are the RMS difference, $R$, the slope of the residuals to the reference series, as well as the long-term trend between the last solar cycle minima in 2008 and 2019. Figure 8 shows the TSI variations reconstructed from RPS Ca II K and red data processed with the various tested methods. The TSI reconstruction from RPS data processed with our method is plotted in Figure 4c.

TSI reconstructed from the images processed with our method scores better than when applying other processing methods tested here, followed by the method by Puiu (2019) with slightly worse RMS differences to the PMOD series. All other processing techniques give worse match to the TSI reference series, with RMS differences increasing to $0.35-0.4 \mathrm{Wm}^{-2}$ instead of $0.17 \mathrm{Wm}^{-2}$ obtained with our method. Interestingly, the long-term change in TSI between the minima in 2008 and 2019 returned by different methods shows opposite signs. Specifically, the trend in TSI determined with the processing following Puiu (2019) and Worden et al. (1998) is negative (decreasing TSI), and is $-1.5 \times 10^{-3} \mathrm{Wm}^{-2} \mathrm{y}^{-1}$ and $-10.4 \times 10^{-3} \mathrm{Wm}^{-2} \mathrm{y}^{-1}$, respectively. The latter is also the steepest trend among all methods. For the other three methods, the trend is positive (increasing TSI) and ranges from $2.0 \times 10^{-5} \mathrm{Wm}^{-2} \mathrm{y}^{-1}$ for the method by Brandt \& Steinegger (1998) to $2.6 \times 10^{-3} \mathrm{Wm}^{-2} \mathrm{y}^{-1}$ for our method.

These tests suggest that for reliable irradiance reconstructions, accurate processing of the images is crucial. Uncertainties of the processing methods might considerably affect the determined long-term trends in the reconstructed TSI.

\section{Summary}

Solar irradiance has been measured from space for over four decades now. Despite significant progress in our understanding of the irradiance variability, its long-term trend is still under debate. To address this question, we have reconstructed the TSI over the period 1996-2020 covering the last two solar cycles.
We have used an empirical model which computes the irradiance changes through a linear regression of solar activity indices. We have followed the approach by Chapman et al. (2013) who used the so-called solar photometric sums as input proxies. The photometric sums were produced from full-disc Ca II K, red, and blue continuum observations acquired with the Precision Solar Photometric Telescope at the INAF Observatory of Rome and Ca II K observations taken with the Cartesian Full-Disk Telescope 2 at the San Fernando Observatory.

We find a good agreement between our reconstructed TSI series and the direct TSI measurements by SOHO/VIRGO and SORCE/TIM, as well as the RMIB and PMOD TSI composites. The agreement is worse with the ACRIM TSI composite. We also find a good agreement with the empirical NRLTSI and EMPIRE models, as well as with the semi-empirical SATIRE-S model, whereby the agreement with SATIRE-S and EMPIRE is somewhat better.

$\mathrm{We}$ find a weak declining trend of $-7.8_{-0.8}^{+4.9} \times$ $10^{-3} \mathrm{Wm}^{-2} \mathrm{y}^{-1}$ between the 1996 and 2008 minima. Between the minima in 2008 and 2019, the trend is $-0.1_{-0.02}^{+0.25} \times$ $10^{-3} \mathrm{Wm}^{-2} \mathrm{y}^{-1}$, i.e. the TSI might have decreased slightly although this change is within the error-bars.

Furthermore, measurements from SORCE/SIM (Harder et al., 2009) and from SOHO/VIRGO (Wehrli et al., 2013) suggested conflicting solar-cycle trends in the irradiance variations in the visible part of the spectrum. Based on the analysis of photometric sum series from SF red and blue observations, Preminger et al. (2002) argued that the irradiance variations in the visible are in anti-phase with the solar cycle, in agreement with the SORCE/SIM measurements. Here we used RP observations in the blue and red continuum to produce series of photometric sums and study the phase of their variations. By carefully analysing the accuracy of the image processing applied to the data, we found a small bias in the QS level of RP red and blue continuum observations of the order of $5 \times 10^{-4}$ in antiphase with the solar cycle. We argue that this bias renders the photometric sum indices rather uncertain, such that the correlation to solar activity can change from being negative to marginally positive after the correction. The amplitude of the variations in the visible is very low and is below the uncertainties, which prevents an assessment of the phase of the variations. Results obtained with alternative techniques are even more uncertain.

This approach to irradiance reconstruction is unfortunately limited to a more recent period only, due to the lack of narrow-band continuum observations before the 1980's. Further modifications to the method, e.g. accounting for sunspots using alternative data, such as white light observations (e.g.Willis et al., 2016; Pal et al., 2020), could allow irradiance reconstructions from properly calibrated historical Ca II K observations, e.g. those from Meudon (Malherbe \& Dalmasse, 2019) or Kodaikanal (Chatzistergos et al., 2019c) observatories, to extend the reconstruction back to 1892 (Chatzistergos et al., 2020a).

Acknowledgements. We thank the observers at the Rome and San Fernando sites for all their work in carrying out the observing programs. We also thank Greg Kopp and the anonymous referee for their valuable comments and suggestions that improved this manuscript. Theodosios Chatzistergos acknowledges funding from the European Union's Horizon 2020 research and Innovation program under grant agreement No 824135 (SOLARNET). This work was supported by the 
Italian MIUR-PRIN grant 2017 "Circumterrestrial Environment: Impact of Sun-Earth Interaction" and by the German Federal Ministry of Education and Research (Project No. 01LG1909C). This research has made use of NASA's Astrophysics Data System. The editor thanks Greg Kopp and an anonymous reviewer for their assistance in evaluating this paper.

\section{References}

Alterman BL, Kasper JC, Leamon RJ, McIntosh SW. 2020. Helium abundance heralds the onset of solar cycle 25. arXiv:2006.04669 [astro-ph, physics:physics]. http://adsabs. harvard.edu/abs/2020arXiv200604669A.

Ball WT, Unruh YC, Krivova NA, Solanki S, Harder JW. 2011. Solar irradiance variability: a six-year comparison between SORCE observations and the SATIRE model. A\&A 530: 71. https://doi.org/10.1051/0004-6361/201016189.

Bolduc C, Charbonneau P, Dumoulin V, Bourqui MS, Crouch AD. 2012. A fast model for the reconstruction of spectral solar irradiance in the near- and mid-ultraviolet. Sol Phys 279: 383-409. https://doi.org/10.1007/s11207-012-0019-4.

Brandt PN, Steinegger M. 1998. On the determination of the quiet-sun center-to-limb variation in Ca II K spectroheliograms. Sol Phys 177(1-2): 287-294. https://doi.org/10.1023/A:1004953032251.

Chapman GA, Cookson AM, Preminger DG. 2012. Comparison of TSI from SORCE TIM with SFO Ground-Based Photometry. Sol Phys 276: 35-41. https://doi.org/10.1007/s11207-011-9867-6.

Chapman GA, Cookson AM, Preminger DG. 2013. Modeling total solar irradiance with San Fernando observatory ground-based photometry: Comparison with ACRIM, PMOD, and RMIB composites. Sol Phys 283: 295-305. https://doi.org/10.1007/ s11207-013-0233-8.

Chatzistergos, T. 2017. Analysis of historical solar observations and long-term changes in solar irradiance. PhD thesis. Uni-edition. ISBN 978-3-944072-55-5. https://ui.adsabs.harvard.edu/abs/ 2017PhDT....259C.

Chatzistergos T, Ermolli I, Solanki SK, Krivova NA. 2016. Exploiting four historical Ca II K spectroheliogram archives. In: Coimbra solar physics meeting ground-based solar observations in the space instrumentation era, Dorotovic I, Fischer CE, Temmer M (Eds.), vol. 504 of Astronomical Society of the Pacific Conference Series, San Francisco, pp. 227-237. ISBN 978-1-58381-892-3. http://www. aspbooks.org/a/volumes/article_details?paper_id=37741.

Chatzistergos T, Ermolli I, Krivova NA, Solanki SK. 2018a. Ca II K spectroheliograms for studies of long-term changes in solar irradiance. In: Long-term datasets for the understanding of solar and stellar magnetic cycles, Banerjee D, Jiang J, Kusano K, Solanki S (Eds.), Vol. 340 of IAU Symposium, Cambridge University Press, Cambridge, UK, pp. 125-128. https://doi.org/ 10.1017/S1743921318001825.

Chatzistergos T, Ermolli I, Solanki SK, Krivova NA. 2018 b. Analysis of full disc Ca II K spectroheliograms - I. Photometric calibration and centre-to-limb variation compensation. A\&A 609: A92. https://doi.org/10.1051/0004-6361/201731511.

Chatzistergos T, Ermolli I, Falco M, Giorgi F, Guglielmino SL, Krivova NA, Romano P, Solanki SK. 2019a. Historical solar Ca II $\mathrm{K}$ observations at the Rome and Catania observatories. Il Nuovo Cimento 42C: 5. https://doi.org/10.1393/ncc/i2019-19005-2.

Chatzistergos T, Ermolli I, Krivova NA, Solanki SK. $2019 \mathrm{~b}$. Analysis of full disc Ca II K spectroheliograms - II. Towards an accurate assessment of long-term variations in plage areas. $A \& A$ 625: A69. https://doi.org/10.1051/0004-6361/201834402.

Chatzistergos T, Ermolli I, Solanki SK, Krivova NA, Banerjee D, Jha BK, Chatterjee S. 2019c. Delving into the historical Ca II K archive from the Kodaikanal Observatory: The potential of the most recent digitized series. Sol Phys 294(10): 145. https://doi.org/ 10.1007/s11207-019-1532-5.

Chatzistergos T, Ermolli I, Krivova NA, Solanki SK. 2020a. Historical solar Ca II K observations at the Kyoto and Sacramento Peak observatories. J Phy Conf Ser 1548: 012007. https://doi.org/ 10.1088/1742-6596/1548/1/012007.

Chatzistergos T, Ermolli I, Krivova NA, Solanki SK, Banerjee D, et al. 2020b. Analysis of full-disc Ca II K spectroheliograms - III Plage area composite series covering 1892-2019. A\&A 639: A88. https://doi.org/10.1051/0004-6361/202037746.

Choudhary DP, Cadavid AC, Cookson A, Chapman GA. 2020. Variability in irradiance and photometric indices during the last two solar cycles. Sol Phys 295(2): 15. https://doi.org/10.1007/ s11207-019-1559-7.

Crouch AD, Charbonneau P, Beaubien G, Paquin-Ricard D. 2008. A Model for the total solar irradiance based on active region decay. Astrophys $J$ 677: 723-741. https://doi.org/10.1086/ 527433.

Dewitte S, Crommelynck D, Joukoff A. 2004. Total solar irradiance observations from DIARAD/VIRGO. J Geophys Res (Space Phys) 109(A02): 102. https://doi.org/10.1029/2002JA009694.

Dewitte S, Nevens S. 2016. The total solar irradiance climate data record. Astrophys J 830: 25. https://doi.org/10.3847/0004-637X/ $830 / 1 / 25$.

Domingo V, Ermolli I, Fox P, Fröhlich C, Haberreiter M, et al. 2009. Solar surface magnetism and irradiance on time scales from days to the 11-year cycle. Space Sci Rev 145(3-4): 337-380. https://doi. org/10.1007/s11214-009-9562-1.

Dudok de Wit T, Kopp G, Fröhlich C, Schöll M. 2017. Methodology to create a new total solar irradiance record: Making a composite out of multiple data records. Geophys Res Lett 44: 1196-1203. https://doi.org/10.1002/2016GL071866.

Egorova T, Schmutz W, Rozanov E, Shapiro AI, Usoskin I, Beer J, Tagirov RV, Peter T. 2018. Revised historical solar irradiance forcing. A\&A 615: A85. https://doi.org/10.1051/0004-6361/201731199.

Ermolli I. 2001. Modelling solar irradiance variations from PSPT full-disk images. Mem Soc Astron Ital 72:545-548. http://adsabs. harvard.edu/abs/2001MmSAI.72.545E.

Ermolli I, Criscuoli S, Centrone M, Giorgi F, Penza V. 2007. Photometric properties of facular features over the activity cycle. A\&A 465: 305-314. https://doi.org/10.1051/0004-6361:20065995.

Ermolli I, Criscuoli S, Giorgi F. 2011. Recent results from optical synoptic observations of the solar atmosphere with ground-based instruments. Contrib Astron Obs Skaln Pleso 41: 73-84. http:// adsabs.harvard.edu/abs/2011CoSka..41...73E.

Ermolli I, Fofi M, Torelli M, Reardon K. 1998. The RISEPSPT telescope operative at the OAR. Mem Soc Astron Ital 69: 631.

Ermolli I, Matthes K, Dudok de Wit T, Krivova NA, Tourpali K, et al. 2013. Recent variability of the solar spectral irradiance and its impact on climate modelling. Atmos Chem Phys 13: 3945 3977. https://doi.org/10.5194/acp-13-3945-2013.

Fligge M, Solanki SK, Unruh YC. 2000. Modelling short-term spectral irradiance variations. Space Sci Rev 94: 139-144. http:// adsabs.harvard.edu/abs/2000SSRv...94..139F.

Floyd LE, Cook JW, Herring LC, Crane PC. 2003. SUSIM'S 11-year observational record of the solar UV irradiance. Adv Space Res 31: 2111-2120. https://doi.org/10.1016/S0273-1177(03)00148-0. 
Fontenla JM, Harder J, Livingston W, Snow M, Woods T. 2011. High-resolution solar spectral irradiance from extreme ultraviolet to far infrared. J Geophys Res (Atmos) 116(20): 108. https://doi. org/10.1029/2011JD016032.

Fontenla JM, Landi E. 2018. Bright network, UVA, and the physical modeling of solar spectral and total irradiance in recent solar cycles. Astrophys J 861(2): 120. https://doi.org/10.3847/15384357/aac388.

Fröhlich C. 2006. Solar irradiance variability since 1978. Revision of the PMOD composite during solar cycle 21. Space Sci Rev 125: 53-65. https://doi.org/10.1007/s11214-006-9046-5.

Fröhlich C, Crommelynck DA, Wehrli C, Anklin M, Dewitte S, Fichot A, Finsterle W, Jiménez A, Chevalier A, Roth H. 1997. In-flight performance of the virgo solar irradiance instruments on soho. Sol Phys 175(2): 267-286. https://doi.org/10.1023/ A:1004929108864.

Georgieva K, Nagovitsyn Y, Kirov B. 2015. Reconstruction of the long term variations of the total solar irradiance from geomagnetic data. Geomagn Aeron 55: 1026-1032. https://doi.org/10.1134/ S0016793215080095.

Haberreiter M, Schöll M, Dudok de Wit T, Kretzschmar M, Misios S, Tourpali K, Schmutz W. 2017. A new observational solar irradiance composite. J Geophys Res (Space Phys) 122: 5910-5930. https://doi.org/10.1002/2016JA023492.

Harder JW, Fontenla JM, Pilewskie P, Richard EC, Woods TN. 2009. Trends in solar spectral irradiance variability in the visible and infrared. Geophys Res Lett 36(7): L0780. https://doi.org/ 10.1029/2008GL036797.

Hathaway DH. 2015. The solar cycle. Living Rev Sol Phys 12: 4. https://doi.org/10.1007/lrsp-2015-4.

Hudson HS, Silva S, Woodard M, Willson RC. 1982. The effects of sunspots on solar irradiance. Sol Phys 76: 211-219. https://doi.org/ 10.1007/BF00170984.

Judge PG, Egeland R, Henry GW. 2020. Sun-like stars shed light on solar climate forcing. Astrophys J 891(1): 96. https://doi.org/ 10.3847/1538-4357/ab72a9.

Judge PG, Lockwood GW, Radick RR, Henry GW, Shapiro AI, Schmutz W, Lindsey C. 2012. Confronting a solar irradiance reconstruction with solar and stellar data. A\&A 544: A88. https://doi.org/10.1051/0004-6361/201218903.

Kopp G. 2016. Magnitudes and timescales of total solar irradiance variability. J Space Weather Space Clim 6: A30. https://doi.org/ $10.1051 /$ swsc/2016025.

Kopp G, Lawrence G. 2005. The Total Irradiance Monitor (TIM): Instrument design. Sol Phys 230(1): 91-109. https://doi.org/ 10.1007/s11207-005-7446-4.

Krivova NA, Solanki SK, Fligge M, Unruh YC. 2003. Reconstruction of solar irradiance variations in cycle 23: Is solar surface magnetism the cause? A\&A 399: L1-L4. https://doi.org/10.1051/ 0004-6361:20030029.

Krivova NA, Solanki SK, Floyd L. 2006. Reconstruction of solar UV irradiance in cycle 23. $A \& A$ 452: 631-639. https://doi.org/ 10.1051/0004-6361:20064809.

Krivova NA, Balmaceda L, Solanki SK. 2007. Reconstruction of solar total irradiance since 1700 from the surface magnetic flux. A\&A 467: 335-346. https://doi.org/10.1051/0004-6361:20066725.

Krivova NA, Vieira LEA, Solanki SK. 2010. Reconstruction of solar spectral irradiance since the Maunder minimum. J Geophys Res (Space Phys) 115: 12112. https://doi.org/10.1029/2010JA015431.

Lean JL. 2018. Estimating solar irradiance since 850 CE. Earth Space Sci 5(4): 133-149. https://doi.org/10.1002/2017EA000357.

Lockwood M, Ball WT. 2020. Placing limits on long-term variations in quiet-Sun irradiance and their contribution to total solar irradiance and solar radiative forcing of climate. Proc $R$ Soc Lond Ser A Math Phys Eng Sci 476(2238): 20200077. https://doi.org/ 10.1098/rspa.2020.0077.

Malherbe J-M, Dalmasse K. 2019. The new 2018 version of the meudon spectroheliograph. Sol Phys 294(5): 52. https://doi.org/ 10.1007/s11207-019-1441-7.

Mauceri S, Pilewskie P, Richard E, Coddington O, Harder J, Woods T. 2018. Revision of the sun's spectral irradiance as measured by SORCE SIM. Sol Phys 293(12): 161. https://doi.org/10.1007/ s11207-018-1379-1.

Mauceri S, Coddington O, Lyles D, Pilewskie P. 2019. Neural network for solar irradiance modeling (NN-SIM). Sol Phy 294(11): 160. https://doi.org/10.1007/s11207-019-1555-y.

Mauceri S, Pilewskie P, Woods T, Béland S, Richard E. 2020. Intercomparing solar spectral irradiance from SORCE SIM. Earth Space Sci 7: e01002. https://doi.org/10.1029/2019EA001002.

Nesme-Ribes E, Meunier N, Collin B. 1996. Fractal analysis of magnetic patterns from Meudon spectroheliograms. A\&A 308: 213-218. http://cdsads.u-strasbg.fr/abs/1996A\%26A...308..213N.

Pal PS, Verma M, Rendtel J, Manrique SJG, Enke H, Denker C. 2020. Solar observatory Einstein Tower: Data release of the digitized solar full-disk photographic plate archive. Astron Nachr 341: 1-13. https://doi.org/10.1002/asna.202013791.

Peck CL, Rast MP. 2015. Photometric trends in the visible solar continuum and their sensitivity to the center-to-limb profile. Astrophys J 808: 192. https://doi.org/10.1088/0004-637X/808/2/192.

Preminger DG, Chapman GA, Cookson AM. 2011. Activitybrightness correlations for the sun and sun-like stars. Astrophys J Lett 739: L45. https://doi.org/10.1088/2041-8205/739/2/L45.

Preminger DG, Walton SR, Chapman GA. 2002. Photometric quantities for solar irradiance modeling. J Geophys Res (Space Phys) 107: 1354. https://doi.org/10.1029/2001JA009169.

Puiu CC. 2019. Modeling solar irradiance variations on timescales from day to solar cycle with ground-based observations. Master's thesis. Sapienza - University of Rome, Rome.

Rottman GJ, Woods TN, McClintock W. 2006. SORCE solar UV irradiance results. Adv Space Res 37(2): 201-208. https://doi.org/ 10.1016/j.asr.2005.02.072.

Shapiro AI, Schmutz W, Rozanov E, Schoell M, Haberreiter M, Shapiro AV, Nyeki S. 2011. A new approach to the long-term reconstruction of the solar irradiance leads to large historical solar forcing. A\&A 529: 67. https://doi.org/10.1051/0004-6361/201016173.

Shapiro AI, Schmutz W, Schoell M, Haberreiter M, Rozanov E. 2010. NLTE solar irradiance modeling with the COSI code. A\&A 517: A48. https://doi.org/10.1051/0004-6361/200913987.

Shapiro AI, Solanki SK, Krivova NA, Cameron RH, Yeo KL, Schmutz WK. 2017. The nature of solar brightness variations. Nat Astron 1: 612-616. https://doi.org/10.1038/s41550-017-0217-y.

Skumanich A, Lean JL, Livingston WC, White OR. 1984. The sun as a star - Three-component analysis of chromospheric variability in the calcium K line. Astrophys J 282: 776-783. https://doi.org/ $10.1086 / 162262$.

Steinhilber F, Beer J, Fröhlich C. 2009. Total solar irradiance during the Holocene. Geophys Res Lett 36: L19704. https://doi.org/ 10.1029/2009GL040142.

Tapping KF, Boteler D, Charbonneau P, Crouch A, Manson A, Paquette H. 2007. Solar magnetic activity and total irradiance since the maunder minimum. Sol Phys 246: 309-326. https://doi. org/10.1007/s11207-007-9047-x.

Tebabal A, Damtie B, Nigussie M, Bires A, Yizengaw E. 2015. Modeling total solar irradiance from PMOD composite using feedforward neural networks. J Atmos Sol-Terr Phys 135: 64-71. https://doi.org/10.1016/j.jastp.2015.10.007. 
Unruh YC, Solanki SK, Fligge M. 1999. The spectral dependence of facular contrast and solar irradiance variations. $A \& A$ 345: 635-642. http://adsabs.harvard.edu/abs/1999A\%26A...345..635U.

Unruh YC, Ball WT, Krivova NA. 2012. Solar irradiance models and measurements: A comparison in the $220-240 \mathrm{~nm}$ wavelength band. Surv Geophys 33: 475-481. https://doi.org/10.1007/s10712011-9166-7.

Vieira LEA, Solanki SK, Krivova NA, Usoskin I. 2011. Evolution of the solar irradiance during the Holocene. $A \& A$ 531: 6 . https://doi. org/10.1051/0004-6361/201015843.

Walton SR, Chapman GA, Cookson AM, Dobias JJ, Preminger DG. 1998. Processing photometric full-disk solar images. Sol Phys 179: 31-42. https://doi.org/10.1023/A:1005070932205.

Wang Y-M, Lean JL, Sheeley NR Jr. 2005. Modeling the Sun's magnetic field and irradiance since 1713. Astrophys $J$ 625: 522-538. https://doi.org/10.1086/429689.

Wehrli C, Schmutz W, Shapiro AI. 2013. Correlation of spectral solar irradiance with solar activity as measured by VIRGO. $A \& A$ 556: L3. https://doi.org/10.1051/0004-6361/201220864.

Willis DM, Wild MN, Appleby GM, Macdonald LT. 2016. The greenwich photo-heliographic results (1874-1885): Observing telescopes, photographic processes, and solar images. Sol Phys 291(9-10): 2553-2586. https://doi.org/10.1007/s11207-016-0894-1. Willson RC. 1997. Total solar irradiance trend during solar cycles 21 and 22. Science 277: 1963-1965. https://doi.org/10.1126/science. 277.5334.1963.
Woods TN, Eparvier FG, Harder J, Snow M. 2018. Decoupling solar variability and instrument trends using the Multiple SameIrradiance-Level (MuSIL) analysis technique. Sol Phys 293: 76. https://doi.org/10.1007/s11207-018-1294-5.

Worden JR, White OR, Woods TN. 1998. Evolution of chromospheric structures derived from $\mathrm{Ca}$ II $\mathrm{K}$ spectroheliograms: Implications for solar ultraviolet irradiance variability. Astrophys J 496: 998. https://doi.org/10.1086/305392.

Wu C-J, Krivova NA, Solanki SK, Usoskin IG. 2018. Solar total and spectral irradiance reconstruction over the last 9000 years. $A \& A$ 620: A120. https://doi.org/10.1051/0004-6361/201832956.

Yeo KL, Krivova NA, Solanki SK, Glassmeier KH. 2014. Reconstruction of total and spectral solar irradiance from 1974 to 2013 based on KPVT, SoHO/MDI, and SDO/HMI observations. A\&A 570: A85. https://doi.org/10.1051/0004-6361/201423628.

Yeo KL, Krivova NA, Solanki SK. 2017a. EMPIRE: A robust empirical reconstruction of solar irradiance variability. J Geophys Res (Space Phys) 122: 3888-3914. https://doi.org/10.1002/ 2016JA023733.

Yeo KL, Solanki SK, Norris CM, Beeck B, Unruh YC, Krivova NA. 2017b. Solar irradiance variability is caused by the magnetic activity on the solar surface. Phys Rev Letters 119: 091102. https://doi.org/10.1103/PhysRevLett.119.091102.

Cite this article as: Chatzistergos T, Ermolli I, Giorgi F, Krivova NA \& Puiu CC. 2020. Modelling solar irradiance from ground-based photometric observations. J. Space Weather Space Clim. 10, 45. https://doi.org/10.1051/swsc/2020047. 